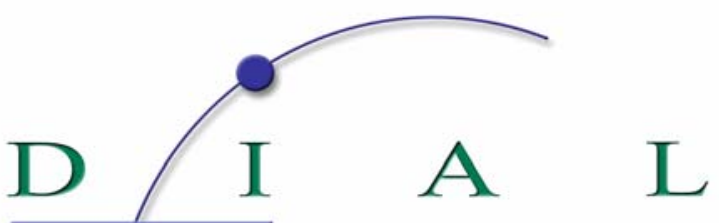

Développement Institutions \& Analyses de Long terme

DOCUMENT DE TRAVAIL

DT/2004/07

\title{
Les pauvres, la démocratie et le Marché : une analyse à partir de trois séries d'enquêtes auprès de la population malgache
}

Mireille RAZAFINDRAKOTO

François ROUBAUD 


\title{
LES PAUVRES, LA DEMOCRATIE ET LE MARCHE : UNE ANALYSE A PARTIR DE TROIS SERIES D'ENQUETES AUPRES DE LA POPULATION MALGACHE
}

\author{
Mireille Razafindrakoto \\ (DIAL - UR CIPRE de l'IRD) \\ razafindrakoto@dial.prd.fr \\ François Roubaud \\ (DIAL - UR CIPRE de l'IRD) \\ roubaud@dial.prd.fr
}

\section{Document de travail DIAL / Unité de Recherche CIPRE}

Septembre 2004

\begin{abstract}
RESUME
Cette étude propose une analyse de l'attitude des pauvres face au processus de double transition politique (démocratisation) et économique (libéralisation) - dans lequel la majorité des pays en développement est actuellement engagée. La thèse la plus couramment avancée postule que les difficultés pour instaurer la démocratie et l'économie de marché résultent de l'attitude de la population, en particulier des pauvres. Les réticences de ces derniers face aux réformes s'expliqueraient par leur faible niveau d'instruction. Nos résultats, basés sur une série de trois enquêtes très détaillées réalisées en 1995, 1998 et 2003 dans la capitale malgache, montrent qu'effectivement, il existe une relation négative entre le niveau de pauvreté et l'adhésion aux principes de l'économie du marché qui ne se réduit pas à un manque d'éducation. L’État, malgré ses déficiences, est considéré comme plus susceptible d'assurer l'objectif d'équité que le marché. En revanche, les pauvres ne se démarquent pas du reste de la population par un moindre soutien aux valeurs démocratiques, ce qui infirme la thèse d'une forme de conservatisme des plus démunis qui se traduirait par une appréhension de la nouveauté ou du changement. Enfin, les exclus de la vie politique et les victimes des dysfonctionnements des institutions tendent à manifester une défiance vis-à-vis de l'État et à se positionner du côté des antidémocrates. La consolidation du processus démocratique est donc conditionnée par l'existence d'institutions étatiques dignes de confiance, efficaces et capables d'assurer l'application effective des principes démocratiques.
\end{abstract}

Mots-clefs : Pauvreté, démocratie, libéralisation, économie de marché, institutions, Madagascar.

\begin{abstract}
This paper presents an analysis of the attitudes of the poor towards the double - political (democratisation) and economic (liberalisation) - transition process in which many developing countries are engaged today. The most common belief to explain the difficulty to implement democracy and market economy relies on the idea that people, especially the poor, have a tendency to resist any reforms. According to this theory, their reluctance is due to their lack of instruction. Our findings, drawn on a series of three detailed surveys conducted in 1995, 1998 and 2003 in the capital of Madagascar, show a negative relationship between the level of poverty and the support to market economy, which is not only conditioned by the deprivation of education. But, on the contrary, the poor are strongly in favour of democracy as the rest of the population. This finding invalidates the hypothesis of a systematic resistance of the poor to change and innovation. The state, in spite of its shortcomings, is considered to be more capable of guaranteeing the equality of opportunities than the market. Finally, those marginalised in politics and the victims of the malfunctions of some institutions tend to have less confidence in the state and to be against democracy. Then, in order to consolidate the democratic process, state institutions must be reinforced to be more reliable, efficient and able to guarantee that the democratic principles are effectively applied.
\end{abstract}

Keywords : Poverty, democracy, liberalisation, market economy, institutions, Madagascar. 


\section{Table des matières}

INTRODUCTION

1. LA POSITION DES PAUVRES VIS-À-VIS DE LA DEMOCRATIE ET DE LA

LIBERALISATION : UNE REVUE DE LITTERATURE.

2. LE CONTEXTE MALGACHE 9

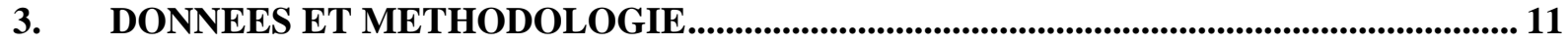

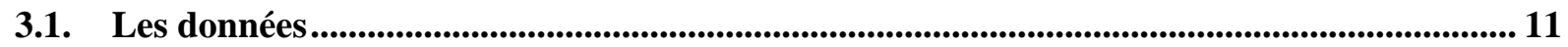

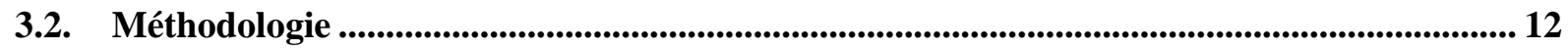

3.2.1. Première étape : construction d'un indicateur synthétique de pauvreté........................................... 12

3.2.2. Deuxième étape : Construction des indicateurs d'adhésion à la démocratie et à l'économie de marché.

3.2.3. Troisième étape : Analyse de la relation entre pauvreté et adhésion aux processus de libéralisation ou de démocratisation

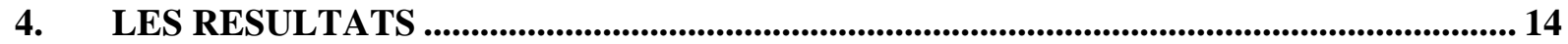

4.1. Les logiques structurant les positions à l'égard des systèmes économique et politique...... 14

4.2. Les pauvres se différencient-ils de l'ensemble de la population ? ........................................... 17

4.3. Quels sont les autres facteurs explicatifs des opinions de la population ? ............................. 21

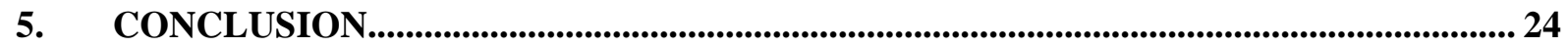

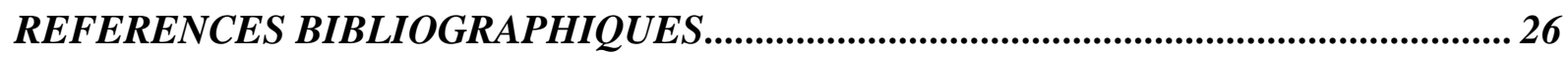

ANNEXES.

\section{Liste des Tableaux}

Tableau 1: $\quad$ Evolution du point de vue des Tananariviens sur la démocratie et la libéralisation................... 10

Tableau 2: $\quad$ Niveau d'instruction en fonction du quartile de revenu en 2002 ............................................. 10

Tableau 3 : $\quad$ Récapitulatif des principaux thèmes abordés dans les modules des trois enquêtes..................... 12

Tableau 4 : $\quad$ Facteurs explicatifs des attitudes à l'égard de la libéralisation et de la démocratie en 1995..... 19

Tableau 5 : $\quad$ Facteurs explicatifs des attitudes vis-à-vis de l'État et de l'ouverture extérieure, 1998 ............. 20

Tableau 6 a : Facteurs associés aux attitudes vis-à-vis de la libéralisation et de la démocratie en 2003..........22

Tableau 6 b : Corrélation de la matrice des résidus du modèle quadrivarié en 2003..................................... 23

Tableau A 1: Matrice des corrélations entre la pauvreté et les positions vis-à-vis de la démocratie et la libéralisation en 1995 .....................................................................................................29

Tableau A 2: Matrice des corrélations entre la pauvreté et les positions vis-à-vis de la libéralisation et l'ouverture extérieure en 1998 ........................................................................................ 29

Tableau A 3 : Matrice des corrélations entre la pauvreté et les positions vis-à-vis de la démocratie et la libéralisation en 2002.

\section{Liste des Graphiques}

Graphique 1: Démocratie et économie de marché. Espace des prises de positions en 1995.

Graphique 2: Démocratie et économie de marché. Espace des prises de positions en 2003.

Graphique 3 : Rôle de l'État et ouverture extérieure. Espace des prises de positions en 1998.

\section{Liste des Annexes}




\section{INTRODUCTION}

La participation de la population, et en particulier celle des pauvres, est aujourd'hui considérée comme une des conditions de réussite des programmes de développement. La mise en œuvre d'un processus participatif pour l'élaboration des Documents Stratégiques de Réduction de la Pauvreté (DSRP) constitue notamment un des principes novateurs de l'initiative lancée fin 1999 par les Institutions de Bretton Woods (Cling, Razafindrakoto et Roubaud, 2002 ; Tungodden, Stern et Kolstad, 2004). Cette initiative qui place la réduction de la pauvreté au centre des nouvelles stratégies de développement préconise la prise en compte du point de vue de la population (Banque mondiale, 2001). Une telle démarche vise à mieux cerner les besoins ainsi que les aspirations et permet d'obtenir l'adhésion des citoyens («ownership ») aux politiques. Notre analyse s'inscrit dans ce cadre en recueillant et en relayant l'opinion des individus dans un pays pauvre, Madagascar. Trois vagues d'enquêtes successives originales ont interrogé la population sur les types de régimes politique et économique qu'elle juge appropriés pour le pays. Cette question ouvre un large débat que nous abordons ici en nous focalisant sur le positionnement des pauvres face au processus de double transition - politique (démocratisation) et économique (libéralisation) - dans lequel la majorité des pays en développement (PED) est actuellement engagée.

Malgré l'accent mis aujourd'hui sur le principe d' «empowerment» et de participation des populations au processus décisionnel, les études qui cherchent à recueillir et à analyser leurs points de vue sur les grandes orientations politiques et économiques dans les pays pauvres sont encore rares. Certes, des avancées existent en la matière. L'initiative de la Banque mondiale visant à recueillir la voix des pauvres a été appliquée dans un certain nombre de pays (Narayan et alii, 2000a et 2000b). Toutefois, cette démarche, qui s'est poursuivie dans le cadre de la préparation des DSRP, donne in fine peu d'informations sur l'appréciation par les populations concernées - en particulier les pauvres - des politiques mises en œuvre. Sont-ils favorables aux réformes de libéralisation économique et à la reformulation du rôle de l'État? Quelles sont leurs positions vis-à-vis des principes démocratiques et de la manière dont ils sont appliqués dans les pays ?

Dans une précédente étude, nous avons mis en évidence la plus grande réticence des pauvres à l'égard de différentes mesures de libéralisation de l'économie dans la capitale malgache (Razafindrakoto et Roubaud, 2002a). Nous avons démontré que l'argument culturel stigmatisant les pauvres en leur attribuant un atavisme « conservateur » pour expliquer leur position ne tient pas. Partant d'une série de trois enquêtes très détaillées réalisées en 1995, 1998 et 2003, portant aussi bien sur les caractéristiques socio-démographiques et économiques des individus que sur leurs aspirations et leurs appréciations des systèmes politiques et économiques, nous nous proposons de poursuivre cette analyse. L'approche adoptée consiste à étudier en parallèle les points de vue des pauvres face à l'instauration de l'économie de marché d'un côté, et de la démocratie de l'autre, ces deux options étant le plus souvent postulées comme devant aller de pair. L'évolution des opinions au fil des années sera observée. Plus spécifiquement, on s'interrogera dans quelle mesure le niveau d'éducation et d'information suffit à expliquer la position des pauvres suivant l'hypothèse classique. Enfin, plus largement, les facteurs explicatifs des opinions sur la libéralisation et la démocratie dans un pays pauvre seront explorés à partir du cas malgache.

L'attention particulière accordée, d'une part, à la définition de la pauvreté en tenant compte de ses multiples dimensions, et d'autre part, à la caractérisation de l'attitude de la population vis-à-vis à la fois des systèmes politique et économique en vigueur constitue un des apports originaux de notre étude. Elle montre que les résultats sur la réticence des pauvres vis-à-vis de la libéralisation économique sont robustes puisqu'on les retrouve pour différentes années. En revanche, contrairement à l'idée que les pauvres seraient plus méfiants face à l'instauration de tout nouveau système, les pauvres ne se démarquent pas du reste de la population concernant le processus de démocratisation. Parallèlement, la thèse expliquant la position des pauvres essentiellement par une moindre capacité à mesurer les enjeux de la libéralisation, compte tenu de leur faible instruction, est battue en brèche. Le niveau d'éducation ou d'information ne suffit pas à expliquer leur opposition à l'économie de marché. Enfin, lorsqu'on explore plus globalement les facteurs structurant les opinions, les exclus de la vie 
politique et les victimes des dysfonctionnements des institutions accordent moins de confiance à l'État et se positionnent plutôt du côté des antidémocrates. Ainsi, la consolidation du processus démocratique doit passer par des réformes visant à rendre les institutions étatiques dignes de confiance, efficaces et capables d'assurer l'application effective des principes démocratiques (en particulier l'égalité devant la loi et la participation de tous à la vie politique).

La première partie de cet article est consacrée à une brève revue de la littérature sur les points de vue de la population sur la démocratie et l'économie de marché ainsi que sur les facteurs expliquant leurs attitudes. La deuxième partie rappelle le contexte qui prévaut à Madagascar en termes de démocratisation et de libéralisation, ainsi que de pauvreté. Les données ainsi que la méthodologie utilisées sont présentées dans la partie suivante. Enfin, les discussions autour des résultats constituent l'objet de la dernière partie.

\section{LA POSITION DES PAUVRES VIS-À-VIS DE LA DEMOCRATIE ET DE LA LIBERALISATION : UNE REVUE DE LITTERATURE}

Le courant aujourd'hui dominant postule que la libéralisation économique et la démocratisation doivent aller de pair pour favoriser la croissance, et plus globalement le développement. Les choix en termes de politique économique et de régime politique qui sont aujourd'hui préconisés dans la grande majorité des pays en développement en témoignent. Pourtant, Dalton et Ong (2002) notent que le système alliant démocratie et libéralisme économique est surtout prééminent dans les pays de l'OCDE 1 . Reprenant l'analyse de Lindblom (1977), ils relèvent que différentes combinaisons de systèmes politiques et économiques ont été adoptées historiquement en Europe. Certains choix alternatifs sont encore actuellement expérimentés dans des pays d'autres continents. Notamment, la "sociale démocratie » qui valorise en même temps l'intervention de l'État dans l'économie et la démocratie - qui était une tradition en Europe - se retrouve dans des pays scandinaves ; tandis que le Chili de Pinochet, la Chine, Singapour et le Vietnam ont allié économie de marché et régime autoritaire, avec des résultats positifs en termes de croissance économique.

Sans entrer dans le vaste débat sur les relations de causalité entre le régime politique et économique d'une part, et la croissance - ou plus largement le développement - d'autre part, la thèse la plus couramment avancée dans la littérature souligne l'existence d'une corrélation positive entre le niveau de richesse d'un pays et l'adhésion aux valeurs démocratiques ainsi qu'à l'économie du marché2. Plusieurs auteurs insistent sur le renforcement mutuel entre les deux processus de libéralisation économique d'un côté et politique de l'autre ${ }^{3}$. Par ailleurs, il est globalement admis que la démocratisation a plus de mal à se concrétiser dans les pays pauvres ${ }^{4}$.

Partant de ces constats globaux, basés pour la plupart sur des analyses transversales, plusieurs études attribuent les mêmes causes aux difficultés de mise en place aussi bien de l'économie de marché que de la démocratie dans les pays pauvres. Trois types de raisons sont le plus souvent invoqués : l'incompatibilité des systèmes de valeurs traditionnelles avec les principes de la démocratie et de l'économie de marché dans les sociétés étudiées ; les mauvaises performances économiques, considérées par la population comme des résultats de la double transition politique et économique, créant des résistances à la poursuite de l'ensemble des réformes; la réticence des pauvres - qui

1 En fait, la démocratie peut prendre différentes formes, les arrangements institutionnels variant selon les pays. Dans cette analyse, nous nous intéressons plus particulièrement au processus de démocratisation. Par souci de simplification, le mot « démocratie » sera utilisé pour désigner l'objectif de respect des principes essentiels de la démocratie et non pour faire référence à un modèle ou à un système institutionnel spécifique.

2 L’articulation entre la démocratie et l'économie de marché est discutée dans Webb et Haggard (1994), Iqbal et You (2001), Banque mondiale (2001 et 2002), Feng (2003). Parmi de nombreuses études Bhagwati (2002) donne un résumé très clair de ce paradigme : "When compared to authoritarian regimes, democracy is more likely to foster an environment that facilitates the innovative and entrepreneurial process so essential for sustained development. On the other hand, democracy is better for development only when accompanied by an expansion of markets and competition. Democracy without markets is unlikely to deliver significant growth. »

$4 \quad$ Voir Przewroski et alii (2000), Feng (2003). 
forment une grande partie de la population - à l'égard de ces deux processus de libéralisation et de démocratisation.

Chacune de ces thèses, que nous développons ci-dessus, se fonde donc essentiellement sur la comparaison des caractéristiques globales de différents pays en fonction de leurs niveaux de richesse et de développement (suivant une approche "inter-pays »). Nous verrons que les études qui portent plus précisément sur l'attitude de la population dans les pays pauvres (suivant une approche que l'on peut qualifier d' « intra-pays ») remettent pour la plupart en question ces théories.

La première thèse postule que les systèmes de valeur prévalant dans les pays en développement ne sont pas compatibles avec les réformes politiques et économiques mises en œuvre (Harrison et Huntington, 2000). Les cultures traditionnelles qui mettraient en avant la primauté de la famille ou de la communauté sur l'individu, ainsi que la prégnance des rapports hiérarchiques suivant les classes d’âges (aînés/cadets) pourrait induire à négliger les libertés individuelles - les libertés politiques mais aussi la liberté d'entreprise. Suivant cette ligne, les démocraties échoueraient dans les pays pauvres faute d'une compréhension de ses principes et de ses avantages, les populations raisonnant essentiellement suivant des logiques ethniques et communautaires in fine à l'encontre de leurs intérêts (Varshney, 1999).

Cette thèse générale est cependant infirmée par différentes études basées sur des enquêtes empiriques détaillées auprès des populations. A titre d'exemple, à Madagascar, la population était déjà majoritairement acquise aux principes démocratiques dès les premières années de la transition politique (Roubaud, 2000). L'analyse montre que les logiques identitaires (ethniques ou communautaires), vues comme des obstacles à la démocratie, ne régissent pas plus les comportements électoraux à Madagascar que dans les pays occidentaux ${ }^{5}$.

Soulignons parallèlement que l'hypothèse invoquant la spécificité des valeurs «asiatiques » pour expliquer les difficultés pour mettre en place la transition démocratique dans beaucoup de pays est également démentie par les enquêtes auprès de la population (Dalton et Ong, 2003).

En fait, plus généralement, lorsqu'on interroge les citoyens des pays d'Afrique - continent où la pauvreté sévit avec le plus d'acuité - ils expriment une nette distinction entre les deux formes de libéralisation (politique et économique) postulées comme allant de pair dans les stratégies de développement prônées par les institutions de Bretton Woods.

D’un côté, la population manifeste effectivement d'importantes réserves à l'égard du processus visant l'instauration de l'économie de marché. C'est notamment le cas des habitants de la capitale à Madagascar (Razafindrakoto et Roubaud, 1996). Pour le Ghana, Bratton, Lewis et Gyimah-Boadi (2000) constatent un soutien très mitigé des réformes impliquant le retrait de l'État. Même en Ouganda où l'économie de marché recueille relativement plus d'opinions favorables, les interventions de l'État dans la sphère économique sont revendiquées (Logan et alii, 2003). Bratton et Mattes (2000) notent dans six pays africains un jugement globalement négatif à l'encontre des programmes d'ajustement structurel dont les principales mesures visent la libéralisation de l'économie.

De l'autre, comme dans le cas malgache, la population ne se caractérise pas par une méfiance à l'égard de la démocratie. Bien au contraire, les enquêtes « afrobaromètre » montrent que les habitants des pays africains revendiquent les valeurs démocratiques (Afrobarometer, 2003). Le soutien partout majoritaire, voire dans certains cas massif (plus de $75 \%$ d'opinions favorables dans quatre des neuf pays étudiés) pour le principe d'« un homme, une voix » n’autorise pas le doute sur ce point.

\footnotetext{
Dans la même lignée, Ramamonjisoa (2002) souligne que la population malgache a refusé de céder aux manipulations ethnicistes de certains politiciens au cours de la crise politique de 2001-2002, cette dernière s'étant finalement résolue de façon pacifiste. L’auteur explique cette attitude des habitants de l'Ile par les valeurs culturelles malgaches prônant systématiquement la négociation, le consensus et le respect des positions de chacun. De telles valeurs peuvent être considérées, selon l'analyse, comme un acquis préparatoire à la démocratie dans la mesure où elles développent la capacité à dialoguer et à trouver en commun des objectifs. Ce constat va aussi à l'encontre de la thèse reposant sur l'incompatibilité des valeurs traditionnelles et de la démocratie.
} 
La deuxième thèse suppose que la population attend de la transition politique et économique des résultats directs et à court terme sur l'amélioration de leur niveau de vie. Suivant cette vision « instrumentale » du double processus de libéralisation et de démocratisation, le soutien accordé ou non par les individus aux réformes découlerait finalement d'un choix rationnel: de mauvaises performances économiques entraînent directement une condamnation du type de régime politique et économique en vigueur. D'où notamment les difficultés pour mener de front la transition politique et économique (Elster, 1993). Certains auteurs considèrent que les réformes en faveur de l'économie de marché, même si elles sont plus efficaces pour la croissance, auraient des effets moins évidents à saisir. Pour cette raison, les populations des pays pauvres favoriseraient le court terme et privilégieraient les programmes avec des impacts directs et immédiats tels que les transferts directs (Varshney, 1999).

Cette thèse qui met en avant l'horizon limité des populations se trouve également invalidée empiriquement lorsqu'on analyse précisément l'évolution des opinions en fonction de la situation économique en Afrique. En introduisant la distinction entre la demande (en référence à une conception idéale de la démocratie) et l'offre de démocratie (telle qu'elle existe) dans douze pays africains, Mattes et Bratton (2003) aboutissent aux constats suivants :

- D’une part, les populations adhèrent aux principes démocratiques. Si la vision " instrumentale » de la démocratie primait, ce soutien pourrait être fragile. La préférence pour ce type de régime serait moins prononcée, voire remise en question, si le processus de démocratisation était sans résultats sur les conditions de vie de la population. Toutefois, les opinions négatives sur les performances des gouvernements élus n'ayant pas d'influence sur le soutien à la démocratie, les auteurs en déduisent que la démocratie est revendiquée pour sa valeur intrinsèque.

- D'autre part, il n’y a pas de confusion sur les critères mis en avant par la population pour exprimer son jugement sur le fonctionnement effectif de la démocratie (l'offre). La démocratie est bien évaluée en fonction de sa capacité à assurer un certain nombre de droits, libertés politiques (élections libres, liberté d'expressions, etc.) et non de façon instrumentale en fonction des résultats économiques du régime en place ou son aptitude à satisfaire les besoins économiques de la population.

Dans la même lignée, en mobilisant les enquêtes auprès de la population en Amérique Latine (Latinobarometro surveys), Graham et Sukhtankar (2002) constatent que la crise économique n'a pas réduit le soutien à la démocratie.

Certes, le lien entre les deux sphères - politique et économique - ne peut être nié. Mais le fait que la population distingue ses attentes vis-à-vis du processus de démocratisation et vis-à-vis de l'instauration de l'économie de marché mérite d'être souligné. Ces résultats mettent également en défaut l'idée selon laquelle une opinion positive sur la libéralisation politique devrait conduire à adopter une attitude en faveur de la libéralisation économique et vice versa ${ }^{6}$. Les discussions restent donc ouvertes quant à l'éventualité d'un renforcement mutuel des points de vue sur les volets politique et économique. Suivant les contextes, des résultats économiques négatifs peuvent mettre en danger le processus de démocratisation, mais des performances économiques positives sont aussi susceptibles de bloquer la transition politique. La peur de l'impact économique d'une éventuelle instabilité politique pourrait en effet l'emporter (Feng, 2003) ${ }^{7}$.

Enfin, la troisième théorie postule que c'est la structure des sociétés qui constitue le principal frein à la mise en place de la démocratie et des réformes économiques. Le raisonnement est tiré d'un double constat : ce sont les plus démunis, les exclus, les plus vulnérables qui sont les plus réfractaires aux changements (Walton et Seddon, 1994 ; Richard, 2000 ; Inglehart, 2000) ; Or ces derniers sont majoritaires dans les pays pauvres. Les difficultés pour instaurer la démocratie et l'économie de marché résulteraient ainsi des effets induits de la pauvreté. La nécessité vitale de satisfaire d’abord les

\footnotetext{
6 Graham et Pettinato (2001) suggèrent, à partir de leur analyse sur les pays d’Amérique Latine, la possibilité d’un renforcement mutuel des opinions favorables à la démocratisation d’une part et à la libéralisation économique d'autre part. Bratton, Lewis et Gyimah-Boadi (2000) abondent également dans ce sens pour le cas du Ghana.

L’exemple de la Tunisie peut être invoqué.
} 
besoins de base relèguerait au second plan des besoins « supérieurs » (libertés politiques, autonomie, etc.). Faute de moyens - de ressources matérielles, mais également de temps et d'éducation - les pauvres se caractériseraient par une moindre prédisposition aux vertus des libertés individuelles: liberté d'entreprendre bien sûr, mais aussi liberté de choisir leurs représentants. Suivant cette ligne d'interprétation, les pauvres seraient plus conservateurs et attachés aux institutions traditionnelles (État, famille) et aux principes moraux qu'elles promeuvent (autorité, discipline, etc.). Les théoriciens du « post-modernisme » semblent bien aller dans ce sens, lorsqu'ils mettent en avant le rôle central de l'éducation dans la diffusion des valeurs individualistes et du libéralisme culturel dans la plupart des démocraties occidentales (Inglehart, 1993, 1997). Cette thèse rejoint la première portant sur la spécificité des valeurs dans les pays pauvres sachant que l'argument culturel est aussi invoqué. Mais c'est la pauvreté qui est clairement désignée comme la source des résistances aux processus de transition politique et économique dans cette troisième thèse.

Notons que les résultats établis par Kaufmann et Kraay (2002), en comparant différents pays en coupe transversale, vont à l'encontre de l'idée que les plus riches seraient mieux disposés à adhérer aux valeurs démocratiques. Ces auteurs montrent une causalité positive de la gouvernance - dont la démocratie est un des éléments constitutifs - sur le niveau de richesse des pays. En revanche, ils trouvent un effet très faible, voire négatif, du revenu par tête sur la qualité de la gouvernance. Ainsi, selon leur analyse (suivant une approche inter-pays), les pays riches ne sont pas plus enclins à adopter un régime démocratique que les pays pauvres. Par extension, en appliquant leur raisonnement au niveau de la population à l'intérieur des pays (approche intra-pays), une des explications qu'ils suggèrent est que les plus riches - les élites notamment - ne cherchent pas particulièrement plus de démocratie car ils bénéficient de privilèges résultant de la détention du pouvoir.

Par ailleurs, Sandbrook (2000) propose une explication du positionnement des pauvres à l'encontre des réformes en faveur de l'économie de marché en insistant sur le fait que ces dernières ont laissé très peu de marges de manœuvre aux pays en termes de politique sociale ou de redistribution. Elles ont accru la vulnérabilité et la marginalisation des pauvres.

Les analyses empiriques basées sur les enquêtes d'opinion auprès de la population confirment que les pauvres affichent une plus grande réticence à l'égard des différentes formes de libéralisation économique ${ }^{8}$. Mais pour le cas de Madagascar, l'argument culturel postulant que les pauvres ont une tendance " conservatrice » est infirmé (Razafindrakoto et Roubaud, 2002a). Les pauvres se montrent notamment relativement plus ouverts en matière de libéralisme politique (revendication de la démocratie et opposition au régime de parti unique) et de libéralisme culturel (changements de religion ou aux mariages inter-ethniques).

Graham et Pettinato (2001) constatent en analysant dix-sept pays latino-américains que les pauvres ont tendance à se positionner à l'encontre de la transition politique comme de la transition économique. A l'inverse, les plus riches seraient relativement plus favorable à la démocratie et à l'économie de marché.

En revanche, en étudiant le cas de douze pays africains, Mattes, Bratton et Davids (2003) montrent que les pauvres ne se caractérisent pas par un moindre soutien à la démocratie. Mattes et Bratton (2003) concluent que les opinions sur la démocratie en Afrique ne s'expliquent pas ou peu par les valeurs culturelles, ni par l'appartenance sociale ou ethnique. Ils mettent en avant l'effet d'apprentissage comme facteur déterminant les attitudes en faveur de la démocratie. Cet effet passerait par plusieurs canaux : l'éducation, les média, la participation à des associations, l’histoire spécifique de la démocratie et ses performances passées dans chaque pays.

En conclusion, les études basées sur des enquêtes d'opinion auprès des populations dans les pays pauvres (en particulier d'Afrique) contredisent dans leur majorité les théories dominantes établies à

8 Ce résultat a été notamment constaté pour le Sri-Lanka (Rannan-Eliya, 1996), pour Madagascar (Razafindrakoto et Roubaud, 1996 et 2002a), pour plusieurs pays d'Amérique Latine (Graham et Pettinato, 2001) et pour différents pays d'Afrique Australe (Bratton et Mattes, 2003). 
partir d'analyses transversales (« inter-pays »). Les citoyens des pays considérés expriment une nette distinction entre les deux formes de libéralisation (politique et économique). S’ils manifestent des réticences à l'égard des réformes de marché, leur adhésion massive aux principes démocratiques ne fait pas de doute. Ce résultat met en défaut la thèse postulant que les valeurs qui prévalent dans les pays pauvres s'opposent à la reconnaissance de toutes formes de libertés individuelles, qu'elles soient politiques ou économiques. La thèse supposant la primauté de la vision « instrumentale » du processus de libéralisation et de démocratisation est également infirmée par l'attachement constant de la population à l'«idéal démocratique » malgré les mauvaises performances économiques. Enfin, les pauvres ne peuvent être stigmatisés comme les principaux réfractaires à la double transition. Ils ne se distinguent pas de leurs concitoyens en termes de soutien aux principes démocratiques.

\section{LE CONTEXTE MALGACHE}

Depuis plus d'une décennie, pour contrecarrer les effets dévastateurs sur la population des choix politiques opérés dans le passé, Madagascar s’est engagé dans un vaste processus de transition économique et politique. Différents fronts ont été ouverts. D’un côté, des réformes de libéralisation de l'économie ont été mises en œuvre avec notamment la privatisation des entreprises para-publiques, la réforme de la fonction publique, l'instauration d'un système de recouvrement des coûts dans les secteurs sociaux ${ }^{9}$. De l'autre, le pays s'est engagé dans la voie démocratique avec des avancées notoires en se dotant progressivement du cadre et des mécanismes institutionnels d'une démocratie formelle. Que ce soit dans le domaine de la liberté d'association, d'expression et des médias ou en matière de processus électoral, Madagascar jouit d'une position relativement enviable. Il s'agit en particulier d'un des rares pays du continent à avoir effectué une triple transition électorale - en 1993, en 1996 et en 2002 - même si la dernière s'est faite à l'issue d'une crise politique majeure qui a paralysé le pays durant plus de six mois.

Toutefois, si un certain nombre de principes semblent acquis en termes de démocratie et plus globalement de gouvernance, leur mise en application sur le terrain est loin d'être évidente. Le processus de démocratisation reste fragile. Les mouvements de contestation de grande ampleur qui ont eu lieu en 1991 et en 2001 découlent d'une insatisfaction de la population relativement à leurs attentes. La trajectoire très favorable de l'économie au moment de ces événements n’a pas suffi au maintien du pouvoir en place. La population a même accepté (à trois reprises 1972, 1991 et 2001) de remettre en cause une dynamique économique ascendante pour dénoncer les inégalités et l'injustice - en particulier la corruption (à grande échelle) et la fraude électorale - et réclamer une meilleure prise en compte de leurs aspirations. La crise politique de 2001-2002, comme celle de 1991, a eu des effets très néfastes du point de vue économique. En même temps, le renversement du pouvoir en place et le règlement pacifique de la crise ont engendré d'immenses espoirs du côté de la population. Leur réalisation dépendra des avancées obtenues sur deux fronts fortement imbriqués : la volonté et la capacité des autorités à opérer de réels changements en termes de gouvernance et de démocratie d'une part ; l'amélioration des conditions de vie de la population d'autre part, dans un pays où l'incidence de la pauvreté est très élevée. A Antananarivo - sur laquelle se concentre cet article - $20 \%$ des habitants vivaient sous le seuil extrême de 1\$PPA par tête et par jour en 2001 (Razafindrakoto et Roubaud, 2002b). Ce pourcentage s’est accru après la crise de 2002.

Si on s'intéresse à l'évolution du point de vue de l'ensemble de la population de la capitale, il semble que la libéralisation économique a gagné en termes de soutien (voir tableau 1). $68 \%$ des Tananariviens se déclaraient favorable à cette option en 2003 alors que le pourcentage correspondant était de $51 \%$ en 1995. De même, les Tananariviens sont plus nombreux à exprimer leur accord à la privatisation de certaines entreprises para-publiques (le pourcentage passe de $46 \%$ à $68 \%$ ). Toutefois, les partisans de l'intervention de l'État dans l'économie constituent toujours un groupe qui est loin d'être minoritaire. Un tiers de la population réclame encore un système d'économie administrée et la moitié se déclare en faveur d’un État jouant un rôle majeur dans la sphère économique.

\footnotetext{
9 Cette politique visant le recouvrement des coûts dans les secteurs de la santé et de l'éducation a été provisoirement abandonné suite à la crise de 2002.
} 
Parallèlement, la part de la population acquise au sentiment démocratique a enregistré un bond en avant. Ceux qui affirment que la démocratie constitue le système le plus favorable pour le pays passe de $65 \%$ en 1995 à $87 \%$ en 2003. Ce résultat remet en question la thèse d'une vision purement instrumentale de la démocratie dans les pays pauvres. La crise politique de 2002 - qui paradoxalement peut être interprétée comme une étape dans le processus de consolidation de la démocratie - a eu un impact économique très négatif sur le bien-être de la population ${ }^{10}$, sans pour autant amoindrir leur adhésion aux principes démocratiques.

Tableau 1: Evolution du point de vue des Tananariviens sur la démocratie et la libéralisation

\begin{tabular}{|c|c|c|c|c|c|c|}
\hline TRANSITIONECONOMIQUE & 1995 & 1998 & 2003 & TRANSITIONPOLITIQUE & 1995 & 2003 \\
\hline -enfaveur libéralisationéconomique & $51 \%$ & & $68 \%$ & -Ladémocratieestlemeilleur ) & & \\
\hline -enfaveuréconomieadministrée & $49 \%$ & & $32 \%$ & systèmepourlepays ～） & $65 \%$ & $87 \%$ \\
\hline -rôlemajeurdel’État dansl'économie & $73 \%$ & & $53 \%$ & & & \\
\hline -placedu secteurprivé,État arbitre & $27 \%$ & & $47 \%$ & -enfaveurdeladémocratie & & $91 \%$ \\
\hline -Pour laprivatisation detoutes lesEPU & $10 \%$ & $14 \%$ & $13 \%$ & & & \\
\hline -Pourlaprivatisation decertaines & $46 \%$ & $62 \%$ & $68 \%$ & -Il fautplus de démocratie & $65 \%$ & \\
\hline -Contre touteprivatisation & $44 \%$ & $24 \%$ & $19 \%$ & & & \\
\hline
\end{tabular}

Sources : Enquêtes 1-2-3, modules thématiques, 1995, 1998, 2003, INSTAT/DIAL, nos propres calculs.

De manière générale, nos résultats confortent les analyses antérieures qui montrent que l'existence d'une demande démocratique dans les pays pauvres ne peut être mis en doute. Malgré les dysfonctionnements de la démocratie dans la pratique, pouvant induire chez certains la revendication d'un certain contrôle, le fait que la population se prononce de plus en plus massivement en faveur de la démocratie dans la capitale malgache remet en question la thèse selon laquelle les populations des pays pauvres ne sont pas particulièrement attachées aux valeurs démocratiques.

Une des thèses que nous testerons dans l'analyse porte sur l'influence de l'éducation sur le point de vue des individus. Sur ce point, il convient de préciser le contexte et de souligner que Madagascar se caractérise par un niveau d'instruction relativement élevé comparé à la moyenne des pays africains. A titre d'illustration, alors que l'on compte $20 \%$ de non scolarisés pour l'ensemble de sept capitales d'Afrique de l'Ouest ${ }^{11}$, ces derniers ne représentent que $2 \%$ à Antananarivo. Une forte corrélation est constatée entre le revenu et l'éducation, mais le recoupement entre la pauvreté monétaire et la faiblesse du capital humain est partiel. Le tableau 2 montre que même pour le quartile le plus pauvre, près de la moitié ont au moins atteint le collège. Le pourcentage correspondant monte à près des deux tiers pour l'ensemble de la population.

Tableau 2: Niveau d'instruction en fonction du quartile de revenu en 2002

\begin{tabular}{|c|c|c|c|c|c|}
\hline En \% & $1^{\mathrm{er}}$ quartile & $2^{\mathrm{e}}$ quartile & $3^{\mathrm{e}}$ quartile & $4^{\mathrm{e}}$ quartile & Total \\
\hline - pas d'instruction & 5,0 & 1,5 & 1,8 & 0,4 & 2,1 \\
\hline - enseignement primaire & 49,6 & 36,0 & 26,2 & 7,3 & 29,5 \\
\hline - $\quad$ collège & 35,2 & 37,9 & 29,2 & 22,5 & 31,1 \\
\hline lycée & 8,9 & 18,8 & 26,4 & 32,6 & 21,8 \\
\hline supérieur & 1,4 & 5,8 & 16,4 & 37,2 & 15,4 \\
\hline
\end{tabular}

Source : Enquêtes 1-2-3, INSTAT/DIAL, 2002, nos propres calculs.

Notes : Quartile de revenu par tête des ménages. Niveau d'instruction des individus de plus de 18 ans.

10 En effet, une chute de $11 \%$ du revenu par tête des ménages a été enregistrée entre la fin de l'année 2001 et la fin de 2002 (Ramilison, 2003).

11 Ce chiffre a été calculé sur la population des plus de 18 ans, à partir des données des enquêtes PARSTAT menées par les Instituts Nationaux de Statistiques et coordonnées par AFRISTAT et DIAL. Les capitales considérées sont : Abidjan, Bamako, Cotonou, Dakar, Lomé, Ouagadougou et Niamey. Les non scolarisés sont ceux qui n’ont fréquenté ni l’école primaire ni l’école coranique. 


\section{DONNEES ET METHODOLOGIE}

\subsection{Les données}

Les données mobilisées dans cette étude proviennent de l'enquête 1-2-3, un instrument de suivi de la pauvreté et de la gouvernance urbaine. Les enquêtes ont été mises en œuvre sur le terrain par l'Institut National de la Statistique (INSTAT). L'enquête 1-2-3 est constituée d'un dispositif de base qui a les caractéristiques d'une enquête statistique classique et qui est réalisé suivant une périodicité annuelle depuis 1995. Il porte notamment sur l'emploi, le secteur informel, les revenus et les conditions de vie des ménages. A cette architecture de base viennent s'ajouter des modules thématiques centrés sur des sujets variables suivant les années.

L'analyse repose plus précisément sur trois séries d'enquêtes représentatives auprès des ménages réalisées dans la capitale malgache en 1995, 1998 et 2002-2003 :

- en 1995, les modules thématiques de l'enquête portaient sur le rôle de l'État dans l'économie, les privatisations ainsi que sur la démocratie. Ces modules ont été administrés auprès de 840 individus tirés de façon aléatoire dans l'échantillon représentatif des ménages de l'enquête de base ;

- en 1998, les thèmes des modules étaient focalisés sur le rôle et le fonctionnement des institutions étatiques ainsi que sur la privatisation et l'ouverture extérieure. L'échantillon enquêté est composé de 2.900 individus.

- en 2002-2003, on dispose d'un questionnaire plus détaillé sur la démocratie, la gouvernance et la pauvreté que nous mobiliserons pour distinguer d'une part l'adhésion aux principes de l'économie de marché ou de la démocratie, et d'autre part, l'appréciation sur le fonctionnement actuel de l'État et de la démocratie. Les données des modules portent sur 2.800 individus.

Les modules thématiques diffèrent d'une année à l'autre, mais on retrouve un certain nombre de questions communes dans les questionnaires des trois enquêtes (voir tableau 3).

L'approche qui consiste à greffer des modules thématiques - qui prennent la forme de sondages d'opinions - sur des enquêtes économiques (quantitatives) classiques présente l'avantage de recueillir à la fois :

- des questions subjectives, propres aux sondages d'opinion (indice de satisfaction, niveau d'adhésion aux politiques, etc.)

- des données objectives sur les comportements et les pratiques sociales (accès aux services publics, participation politique, incidence de la corruption, etc.)

- les caractéristiques socio-économiques classiques (sexe, âge, scolarité, migration, emploi, revenu, consommation, etc.).

La richesse des données - qui combinent des variables économiques standards et des variables d'opinions - offre la possibilité de désagréger les informations suivant les catégories de ménages ou d'individus. L'attention particulière accordée à l'échantillonnage permet de répondre au souci de représentativité et de fiabilité des opinions recueillies. Les enquêtes sont menées sur des échantillons de grande taille constitués suivant un tirage aléatoire ${ }^{12}$. Enfin, par rapport aux études usuelles sur les perceptions ou opinions de la population, on dispose d'une mesure plus précise et plus fiable des revenus et plus généralement du niveau de vie des individus ou ménages. L'enquête 1-2-3 est en effet destinée en premier lieu à mesurer de façon détaillée le niveau et l'évolution des conditions de vie économiques des individus et des ménages.

12 Soulignons que dans la majorité des cas, les enquêtes d'opinion ont recours à la méthode des quotas. La constitution d'échantillons suivant un tirage aléatoire permet un calcul précis des erreurs d'échantillonnage. 
Tableau 3 : Récapitulatif des principaux thèmes abordés dans les modules des trois enquêtes

\begin{tabular}{|c|c|c|c|}
\hline & Enquête 1995 & Enquête 1998 & Enquête 2003 \\
\hline $\begin{array}{l}\text { Libéralisation économique et rôle de l'Etat } \\
\text { - Libéralisation économique vs économie administrée } \\
\text { - Privatisation } \\
\text { - Rôle de l'Etat dans le système éducatif } \\
\text { Appréciation de l'administration et de l'Etat } \\
\text { - Confiance à l'égard de l'administration } \\
\text { - Efficacité de l'administration } \\
\text { - Opinions sur les fonctionnaires } \\
\text { - Qualité de la gestion des entreprises publiques } \\
\text { - Efficacité des opérateurs privés } \\
\text { Ouverture extérieure } \\
\text { - Poids des intérêts étrangers } \\
\text { - Possibilité ou non d'achat de terrain par les } \\
\text { étrangers }\end{array}$ & $\begin{array}{l}X \\
X\end{array}$ & $\begin{array}{l}X \\
X \\
X \\
X \\
X\end{array}$ & $\begin{array}{l}X \\
X \\
X\end{array}$ \\
\hline $\begin{array}{l}\text { Démocratisation } \\
\text { - Adhésion aux principes démocratiques } \\
\text { - Appréciation des différents régimes politiques } \\
\text { - Fonctionnement de la démocratie dans le pays }\end{array}$ & $\begin{array}{l}X \\
X\end{array}$ & & $\begin{array}{l}X \\
X \\
X\end{array}$ \\
\hline
\end{tabular}

\subsection{Méthodologie}

Deux types de techniques sont utilisés ici pour interpréter les résultats : l'analyse en composantes multiples et les estimations économétriques. La méthodologie adoptée comporte trois étapes :

- la première étape consiste à construire un indicateur de pauvreté (suivant une échelle continue) - tenant compte de ses multiples dimensions - et qui se rapporte à chaque individu ;

- la deuxième étape porte sur l'élaboration d'indicateurs donnant la position des individus suivant leur degré d'adhésion aux différentes options (échelles d'adhésion à la libéralisation, à la démocratie ou à l'ouverture extérieure ; échelles de satisfaction concernant l'efficacité de l'État ou sur le fonctionnement actuel de la démocratie) ;

- La troisième étape recourt aux méthodes économétriques pour tester l'impact de la pauvreté (dans ses multiples dimensions) sur les prises de position en matières d'options économiques et politiques.

\subsubsection{Première étape : construction d’un indicateur synthétique de pauvreté}

On part du constat unanimement accepté que la pauvreté a de multiples dimensions. Les différentes approches de la pauvreté (monétaire, conditions d'existence, capital humain, capital social et pauvreté subjective) se recoupent, témoignant qu'il s'agit bien d'un seul phénomène, mais le recoupement est partiel (Razafindrakoto et Roubaud, 2004). Par conséquent, quel critère retenir pour définir la population pauvre ? L'idée est d'éviter une approche normative en accordant d'emblée une importance particulière à une forme de pauvreté relativement aux autres. Nous avons donc considéré quatre types de variables caractérisant chacune une dimension de la pauvreté :

- le niveau de revenu (un flux), suivant l'approche monétaire classique ;

- le niveau de patrimoine (un stock) : la variable retenue est un indicateur synthétique de la possession ou non de différents éléments de patrimoine (voiture, radio, TV, réfrigérateur, cuisinière, etc.) - une approche qui a notamment été adoptée par Sahn et Stifel (2000) our comparer les niveaux de pauvreté dans plusieurs pays d'Afrique ; 
- les conditions d'habitation et d'accès aux services publics : la variable est construite en calculant un «score de privations » ou score cumulatif portant sur les caractéristiques du lieu d'habitation (maisons en dur ou non, nombre de pièces, accès à l'eau, à l'électricité, caractéristiques des toilettes). Cet indicateur a été utilisé entre autres par Lollivier et Verger (1997) pour étudier les différentes facettes de la pauvreté en France ;

- la pauvreté subjective avec deux variables qui sont disponibles uniquement en 2003 : perception générale du niveau de vie et perception de la situation financière du ménage. Cette approche a été adoptée entre autres par Ravallion et Lokshin (1998) dans leurs analyses sur la Russie.

A partir de ces différentes variables, nous avons procédé à une analyse des correspondances multiples $(\mathrm{ACM})^{13}$. On retient le premier axe qui résume le mieux les dimensions correspondant aux quatre critères retenus pour définir la pauvreté (cet axe représente respectivement $22 \%$ de l'inertie globale en 1995, $21 \%$ en 1998 et $19 \%$ en 2003). Il fournit une échelle intégrant les multiples dimensions de la pauvreté. Pour chacune des différentes dimensions (revenu, patrimoine, logement, pauvreté subjective), les variables (quartiles ou modalités) se projettent sur l'axe dans l'ordre, allant des plus défavorisés aux plus favorisés. Les coordonnées des individus sur cet axe forment une variable continue caractérisant le niveau de pauvreté (ou de richesse) multidimensionnelle de leur ménage respectif.

3.2.2. Deuxième étape : Construction des indicateurs d'adhésion à la démocratie et à l'économie de marché.

Un premier éclairage sur le mode de structuration des opinions sur la démocratie et l'économie de marché est fourni par la visualisation de leur représentation dans l'espace. En recourant une nouvelle fois à l'ACM, nous avons cherché à positionner les différentes modalités sur la perception et les attentes de la population concernant le type de régime politique adéquat d'une part et les grandes lignes des options économiques d'autre part.

On retient selon les cas le premier axe ou le premier plan (formé par les deux premiers axes) qui explique le mieux le nuage des prises de position, donc discrimine le mieux la population. Les coordonnées des individus sur chaque axe donnent un indicateur synthétique. Etant donné que différentes questions posées lors de l'enquête se recoupent ou se complètent, cet indicateur résume les points de vue exprimés sur un thème donné (adhésion ou satisfaction à l'égard de la démocratie ; adhésion à la libéralisation; confiance vis-à-vis de l'État). Il représente le positionnement de l'individu relativement à la population étudiée. L'intérêt d'une telle approche - qui ne repose pas sur une mais sur plusieurs questions - est de remédier à l'éventuel caractère normatif des questions posées ou à d'éventuelles interprétations erronées des questions par les personnes interrogées. Cette procédure permet donc d'atténuer les erreurs de mesure.

\subsubsection{Troisième étape: Analyse de la relation entre pauvreté et adhésion aux processus de} libéralisation ou de démocratisation

L'objectif est de caractériser de façon précise les groupes qui se différencient de par leurs attitudes à l'égard du processus de démocratisation d'une part et de libéralisation économique d'autre part. En recourant aux méthodes économétriques et en mobilisant les indicateurs construits dans la deuxième étape, les facteurs explicatifs du niveau d'adhésion ou de satisfaction sont explorés. L'interrogation principale sera dans quelle mesure les pauvres (identifiés à partir de la variable synthétique construite dans la première étape) se distinguent du reste de la population sur ces questions. L'analyse permettra d'étudier en corollaire de façon plus large le mode de structuration des opinions dans un pays pauvre comme Madagascar.

Afin de tester en parallèle l'hypothèse selon laquelle l'économie de marché et la démocratie vont de pair, nous avons choisi d'adopter des modèles de régression bivariée ou quadrivariée (avec un

13 Les variables mobilisées pour l'ACM sont : soit les quartiles (de revenu, de niveau de patrimoine, de la qualité du logement), soit les modalités fournies par le questionnaire (pour la pauvreté subjective). 
traitement simultané des équations sur la libéralisation et la démocratie). Ce type d'estimation permet de vérifier si certaines caractéristiques des individus, en particulier celles non identifiées (inobservables), jouent conjointement sur les points de vue sur les systèmes politique et économique ou si les opinions sur les deux options se construisent de façon indépendante.

\section{LES RESULTATS}

\subsection{Les logiques structurant les positions à l'égard des systèmes économique et politique}

Lorsqu'on explore à partir d'une analyse en composantes multiples (ACM) la manière dont s'organise les opinions sur la démocratie et la libéralisation économique en 1995, le premier plan factoriel (formé par les deux premiers axes représentant respectivement $29 \%$ et $25 \%$ de l'inertie totale) montre une polarisation explicite des opinions ${ }^{14}$. Quatre groupes se différencient (voir graphique 1). D'abord, suivant une première direction (flèche de gauche à droite et de bas en haut), s'opposent ceux qui revendiquent l'intervention de l'État dans l'économie et ceux en faveur du «moins d'État». Les positions sont cohérentes dans la mesure où les individus en faveur de la libéralisation sont aussi ceux qui demandent à l'État de jouer un rôle de simple arbitre et qui se prononcent pour la privatisation des entreprises publiques. Ensuite, les individus se positionnent sur une seconde direction (de gauche à droite mais de haut en bas) en fonction de leur opinion sur la démocratie. Cet axe isole ceux qui déclarent leur opposition à l'instauration d'un régime démocratique de ceux qui lui sont favorables ${ }^{15}$.

\section{Graphique 1 : Démocratie et économie de marché. Espace des prises de positions en 1995}

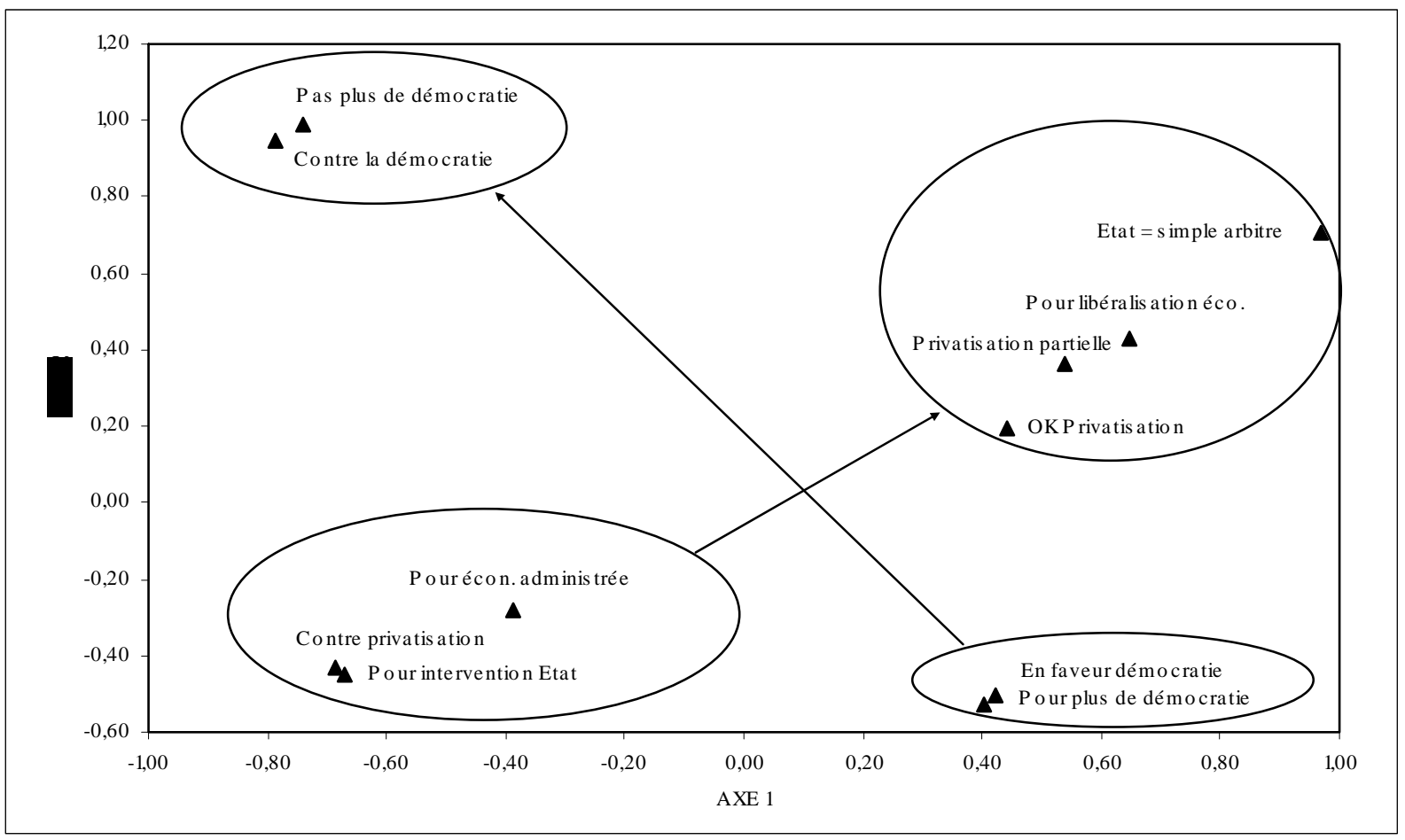

Source : Enquête 1-2-3, 1995, nos propres calculs.

14 Voir en annexe la liste des variables mobilisées pour l'ACM.

15 L’identification des quatre groupes bien spécifiques, avec un axe « politique » quasiment orthogonal à l’axe « économique », témoigne d'une certaine indépendance entre les opinions à l'égard du processus de démocratisation et celles vis-à-vis de la libéralisation. Le système d'équations simultanées présenté dans la suite de l'analyse permettra de tester plus formellement ce résultat. 


\section{Graphique 2 : Démocratie et économie de marché. Espace des prises de positions en 2003}

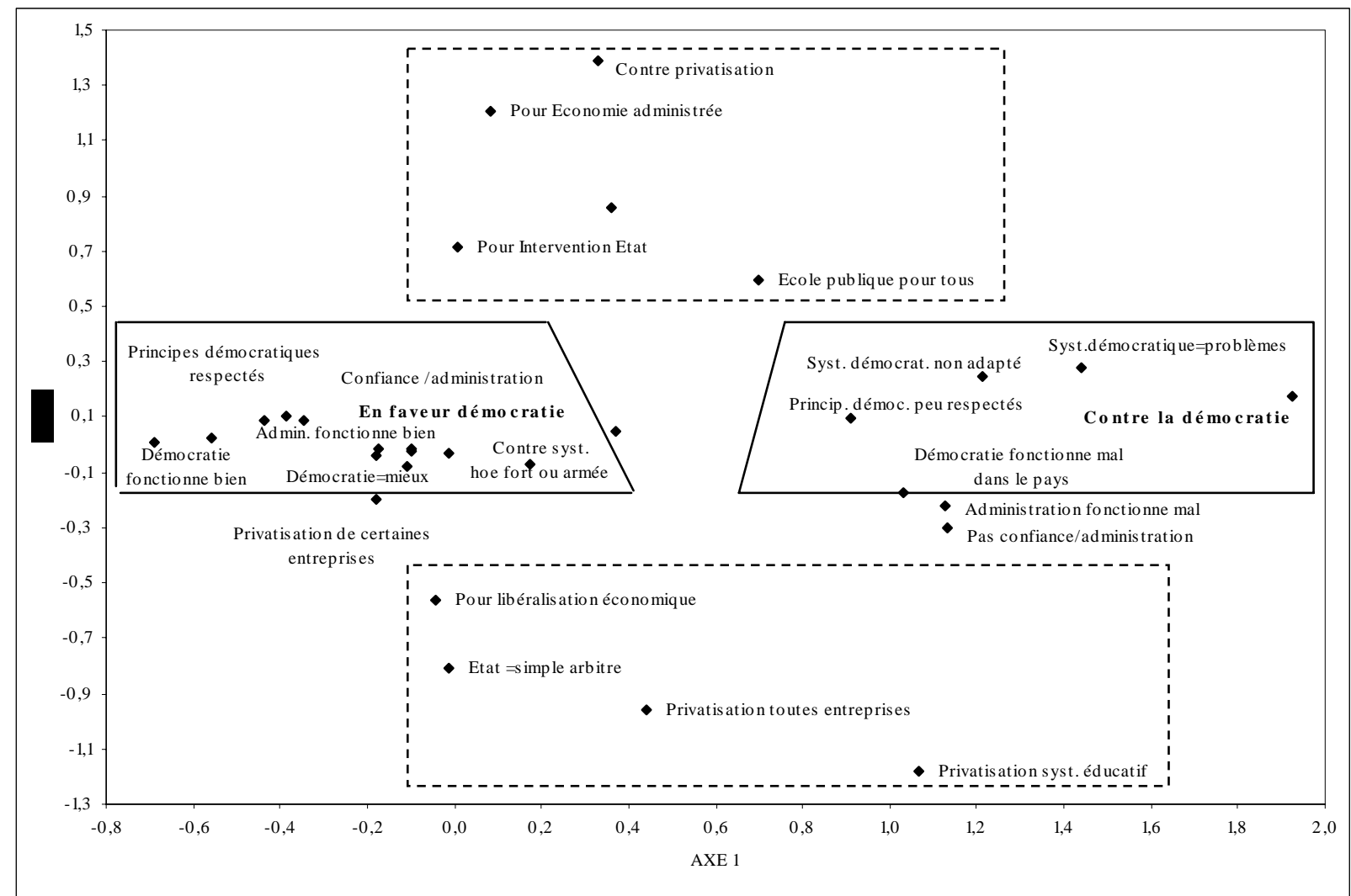

Source : Enquête 1-2-3, 2002-03, nos propres calculs.

Note : Pour une meilleure clarté du graphique, nous n’avons représenté que les variables les plus significatives du point de vue de leurs contributions aux axes factoriels.

Cette configuration de l'espace des prises de positions est robuste puisqu'on retrouve en 2003 l'opposition entre ces quatre groupes en appliquant la même méthodologie mais en mobilisant des questions plus détaillées sur les mêmes thèmes (voir graphique 2$)^{16}$.

Toutefois, l'interprétation des axes fournis directement par ces ACM s'avère délicate sachant que les deux axes opposent les opinions aussi bien sur la transition politique que sur la libéralisation économique. Pour aller plus loin dans l'analyse, pour les deux années 1995 et 2003 pour lesquelles nous disposons à la fois des opinions sur la démocratie et sur la libéralisation économique, nous avons construit de façon séparée les indicateurs concernant l'espace politique d'une part et l'espace économique d'autre part.

Ainsi, pour 1995, nous avons retenu le premier axe de l'ACM restreinte aux questions politiques (cet axe oppose les partisans et les détracteurs du système démocratique). De même, l'ACM portant sur les questions économiques distingue sans surprise sur le premier axe ceux qui sont favorables à la libéralisation et ceux qui lui sont réticents. On dispose donc de deux échelles des attitudes (politiques et économiques) ainsi que des positions (coordonnées sur l’axe) des individus sur ces échelles.

Partant d'un questionnaire plus complet en 2003, les deux premiers axes dans chacun des deux espaces (politique et économique) méritent d'être retenus. Dans chaque cas, le premier porte sur l'adhésion aux principes ou aux options idéologiques (la «demande »). Le second concerne l'appréciation du mode de fonctionnement effectif des principes ou options dans le pays (l'« offre »).

16 La similitude entre les deux configurations des espaces en 1995 et 2003 est encore plus nette si on ne garde en 2003 que les questions posées en 1995 sur l'adhésion aux options (la démocratie et la libéralisation économique) et en excluant celles sur l'évaluation du fonctionnement des options. 
En retenant les questions sur le thème de la démocratie :

- le premier axe (22\% de l'inertie totale) oppose les partisans aux adversaires de la démocratie dans ses principes. Il permet de construire un indicateur du degré d'adhésion aux principes démocratiques ;

- le second axe (14\% de l'inertie) oppose ceux qui sont satisfaits du fonctionnement de la démocratie à ceux plus critiques. Les coordonnées des individus sur cet axe synthétise ainsi l’appréciation sur l’offre de démocratie dans le pays.

En étudiant les attitudes à l'égard de la libéralisation économique :

- le premier axe (19 \% de l’inertie totale) oppose les partisans à la libéralisation économique à ses détracteurs. On en déduit un indicateur synthétique du soutien à la transition économique ;

- le second axe (18\% de l'inertie) oppose ceux qui font confiance aux différentes institutions publiques à ceux méfiants à leur égard. La variable synthétique qui en est déduite porte sur le jugement du fonctionnement des institutions ;

Pour l'année 1998, le questionnaire est centré sur la réforme de la fonction publique et les privatisations. La façon dont les opinions s'organisent sur les deux axes peut également être interprétée aisément, témoignant de la cohérence des réponses données par les personnes enquêtées.

La principale opposition révélée par l'analyse (voir graphique 3) se décline suivant le degré de satisfaction à l'égard du secteur public (sur le premier axe qui représente $16 \%$ de l'inertie totale). D’un côté, le satisfecit est sans réserve avec la manifestation d'une confiance vis-à-vis des institutions étatiques, un jugement favorable sur l'efficacité de l'administration. Ce groupe se caractérise aussi par le soutien accordé aux entreprises publiques et l'opposition aux mesures de privatisation. De l'autre côté, les points de vue manifestent l'absence de confiance à l'égard du service public, une appréciation négative sur son efficacité, son rendement et l'assiduité de fonctionnaires. Parallèlement, ces derniers sont favorables aux privatisations en étant persuadés de la plus grande efficacité des opérateurs privés.

Le second axe (qui représente $10 \%$ de l'inertie globale) porte sur le degré d'adhésion aux réformes d'ouverture extérieure. D’un côté, on retrouve ceux qui sont plutôt réticents à l'égard des privatisations et qui expriment de plus une peur d'une mainmise des étrangers sur l'économie. Ils s'opposent à l'accroissement du nombre ou du poids économiques des étrangers dans le pays. Ils se déclarent contre l'achat de terrain par ces derniers, et même contre la possibilité de bail emphytéotique ${ }^{17}$. Enfin, ils estiment que la priorité devrait être donnée aux opérateurs nationaux dans les cas de privatisation. De l'autre côté, les opinions tendent à être favorables à la privatisation et à l'investissement étranger.

17 Le bail emphytéotique, instauré en août 1996, permet la location de terrain sur une très longue durée (allant jusqu’à 99 ans). Cette option vise à sécuriser les investissements sachant que les étrangers n’ont pas le droit d'acheter des terrains à Madagascar. 


\section{Graphique 3 : Rôle de l’État et ouverture extérieure. Espace des prises de positions en 1998}

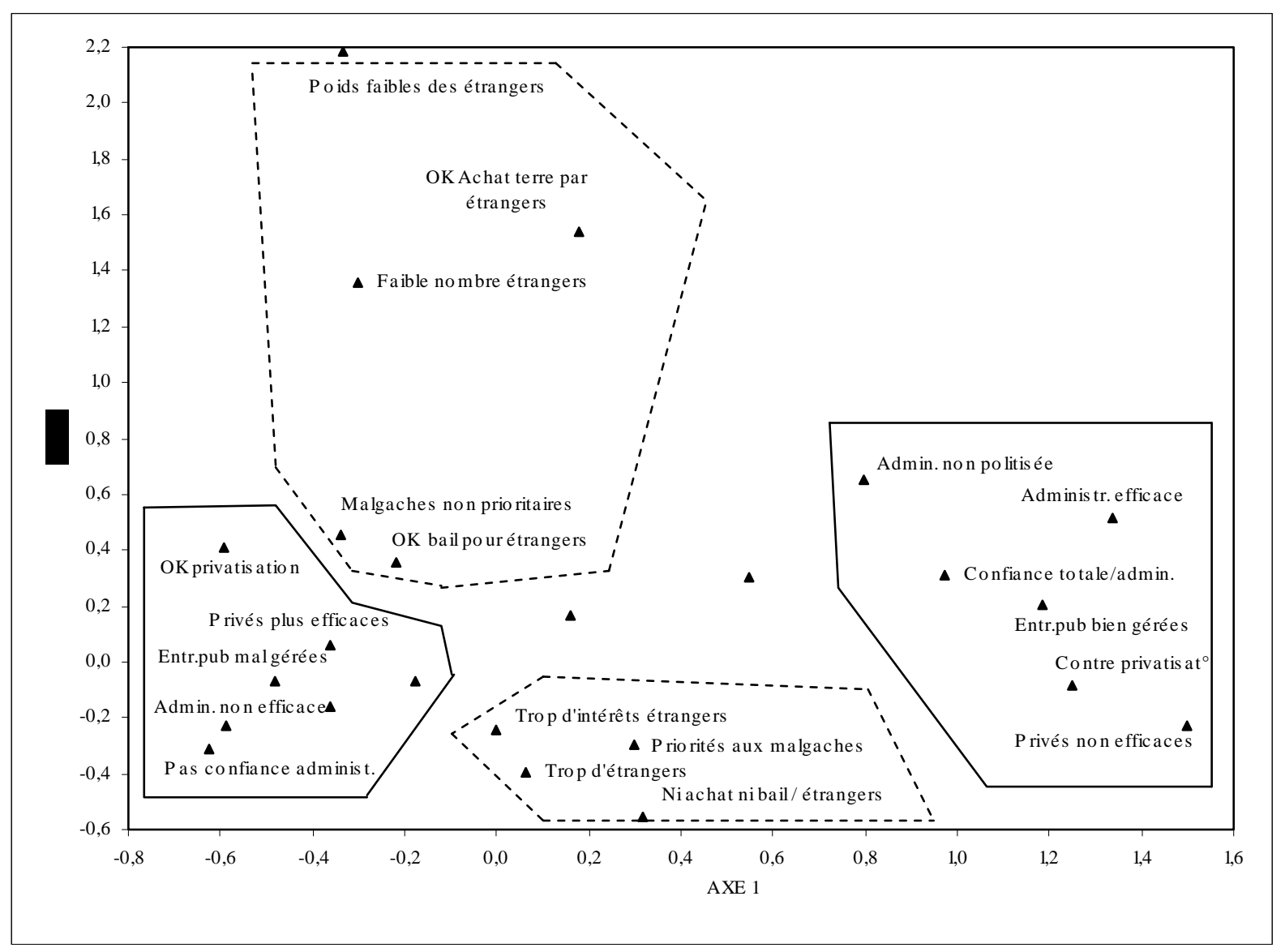

Source : Enquête 1-2-3, 1998, nos propres calculs.

Note: Pour une meilleure clarté du graphique, nous n’avons représenté que les variables les plus significatives du point de vue de leurs contributions aux axes factoriels.

\subsection{Les pauvres se différencient-ils de l'ensemble de la population ?}

L'étape suivante consiste à mobiliser les techniques économétriques afin d'évaluer dans quelle mesure les pauvres ont une attitude qui les différencie du reste de la population. On mobilisera les indicateurs synthétiques construits à partir des coordonnées factorielles des individus sur les différents axes identifiés précédemment. On dispose donc de variables continues sur les attitudes (des moins favorables aux plus favorables) vis-à-vis de chacune des deux processus - de démocratisation et de libéralisation économique.

Une analyse préalable des corrélations entre la pauvreté et les points de vue des individus révèle un lien négatif significatif entre l'adhésion aux principes de l'économie de marché et à l'ouverture économique, d'une part, et le niveau de pauvreté d'autre part (voir tableaux A1, A2 et A3 en annexe). Conformément aux résultats que nous avions obtenus à partir de simples tableaux croisés (Razafindrakoto et Roubaud, 2002a), les pauvres manifestent une méfiance à l'encontre de la libéralisation économique. Parallèlement, la corrélation entre la pauvreté et l'attachement aux valeurs de la démocratie est inexistante en 1995, et négative mais très faible $(-0,04)$ en 2003 . Sachant que la pauvreté et le niveau d'éducation sont fortement corrélés (avec des coefficients allant de -0,43 en 1995 à -0,58 en 2002), on commencera par tester le modèle standard qui postule que le degré d'instruction 
ou d'information suffit à expliquer les positions des pauvres avant d'explorer d'autres facteurs explicatifs ${ }^{18}$.

En premier lieu, pour les trois années considérées (voir tableaux 4, 5 et 6), l'influence positive du niveau d'éducation et du degré d'information (caractérisé par le suivi régulier des informations, la connaissance des réformes de la fonction publique et des mesures de privatisation) sur l'adhésion à la libéralisation économique est clairement établie. Ce résultat conforte la thèse du rôle de l'instruction pour favoriser l'adhésion aux réformes. Cependant, la position des pauvres ne peut s'interpréter comme un simple effet du niveau d'éducation. Même si la faiblesse du capital humain joue négativement sur l'attitude à l'égard de la libéralisation, l'effet ne transite pas uniquement par ce canal. A niveau d'éducation donné, d'autres dimensions de la pauvreté (monétaire, conditions d'existence, etc.) agissent sur les prises de position, les plus démunis exprimant de fortes attentes à l'égard de l'État et s'opposant à son désengagement. Ces derniers se caractérisent aussi par leur appréhension face à l’ouverture extérieure du pays.

En second lieu, les décideurs ont tendance à ne pas tenir compte du point de vue des pauvres pour la définition des orientations politiques. Ils invoquent comme argument le fait que la réticence de ces derniers aux réformes - qu'elles soient économiques ou politiques - résulte d'une faible éducation et d'une forme de conservatisme. Toutefois, la thèse postulant une certaine frilosité des plus démunis à l'égard de tout ce qui est nouveau est démentie par la position des pauvres vis-à-vis du processus de démocratisation. Malgré les contraintes quotidiennes qui pourraient les amener à être moins sensible aux aspirations démocratiques, ils ne se distinguent pas du reste de la population. Dans aucun des modèles estimés, le coefficient de l'échelle de pauvreté n'est significatif. Le soutien accordé par les pauvres à la démocratie est tout aussi massif que celui de l'ensemble des habitants de la capitale.

18 La variable considérée pour le niveau d'éducation est le nombre d'années d'étude réussies rapporté au nombre d’années maximum que l'individu aurait pu atteindre compte tenu de son âge. Par exemple, pour un individu âgé de 18 ans, le maximum d'années d'études potentielles est de 12, l'âge théorique d'entrée à l'école étant de 6 ans. Pour les adultes de plus de 28 ans, le nombre maximum est de 22 ans, ce qui correspond au nombre d'années d'études pour atteindre le niveau universitaire le plus élevé (le doctorat). 
Tableau 4 : Facteurs explicatifs des attitudes à l'égard de la libéralisation et de la démocratie en 1995

Modèle bivarié (régression en équations simultanées)

\begin{tabular}{|c|c|c|c|c|c|c|}
\hline & $\begin{array}{l}\text { En faveur } \\
\text { libéralisation } \\
\text { économique }\end{array}$ & $\begin{array}{c}\text { (1) } \\
\text { En faveur } \\
\text { démocratie }\end{array}$ & $\begin{array}{l}\text { En faveur } \\
\text { libéralisation } \\
\text { économique }\end{array}$ & $\begin{array}{c}(2) \\
\text { En faveur } \\
\text { démocratie }\end{array}$ & $\begin{array}{l}\text { En faveur } \\
\text { libéralisation } \\
\text { économique }\end{array}$ & $\begin{array}{c}\text { (3) } \\
\text { En faveur } \\
\text { démocratie }\end{array}$ \\
\hline $\begin{array}{l}\text { Echelle continue de } \\
\text { pauvreté }\end{array}$ & $\begin{array}{c}-0,19 \\
(5,68)^{* * *}\end{array}$ & $\begin{array}{c}0,01 \\
(0,35)\end{array}$ & $\begin{array}{c}-0,11 \\
(3,00)^{* * *}\end{array}$ & $\begin{array}{c}0,05 \\
(1,14)\end{array}$ & $\begin{array}{c}-0,11 \\
(2,79)^{* * *}\end{array}$ & $\begin{array}{c}0,05 \\
(1,16)\end{array}$ \\
\hline Niveau d'éducation & & & $\begin{array}{c}0,45 \\
(3,42)^{* * *}\end{array}$ & $\begin{array}{c}0,17 \\
(1,04)\end{array}$ & $\begin{array}{c}0,44 \\
(3,38)^{* * *}\end{array}$ & $\begin{array}{c}0,16 \\
(0,96)\end{array}$ \\
\hline $\begin{array}{l}\text { Ne suivent pas les } \\
\text { nouvelles }\end{array}$ & & & $\begin{array}{c}-0,21 \\
(2,07)^{* *}\end{array}$ & $\begin{array}{l}-0,19 \\
(1,54)\end{array}$ & $\begin{array}{c}-0,22 \\
(2,17)^{* *}\end{array}$ & $\begin{array}{l}-0,20 \\
(1,58)\end{array}$ \\
\hline $\begin{array}{c}\text { Pensent que dégradation } \\
\text { situation économique }\end{array}$ & & & & & $\begin{array}{l}-0,06 \\
(0,94)\end{array}$ & $\begin{array}{l}-0,05 \\
(0,70)\end{array}$ \\
\hline Victime corruption & & & & & $\begin{array}{l}-0,03 \\
(0,51)\end{array}$ & $\begin{array}{l}-0,02 \\
(0,26)\end{array}$ \\
\hline $\begin{array}{l}\text { Pensent inégalités }= \\
\text { problème majeur }\end{array}$ & & & & & $\begin{array}{c}0,06 \\
(0,82)\end{array}$ & $\begin{array}{c}0,13 \\
(1,58)\end{array}$ \\
\hline $\begin{array}{l}\text { Pensent problème }= \\
\text { pays étrangers }\end{array}$ & & & & & $\begin{array}{c}-0,16 \\
(1,22)\end{array}$ & $\begin{array}{c}0,14 \\
(0,86)\end{array}$ \\
\hline $\begin{array}{l}\text { Pensent problème }= \\
\text { mentalité population }\end{array}$ & & & & & $\begin{array}{c}0,06 \\
(0,91)\end{array}$ & $\begin{array}{c}0,11 \\
(1,41)\end{array}$ \\
\hline Ménages informels & $\begin{array}{l}-0,08 \\
(1,43)\end{array}$ & $\begin{array}{l}-0,02 \\
(0,25)\end{array}$ & $\begin{array}{l}-0,04 \\
(0,71)\end{array}$ & $\begin{array}{c}0,00 \\
(0,05)\end{array}$ & $\begin{array}{l}-0,04 \\
(0,75)\end{array}$ & $\begin{array}{c}0,00 \\
(0,02)\end{array}$ \\
\hline Chômeurs & $\begin{array}{l}-0,03 \\
(0,22)\end{array}$ & $\begin{array}{l}-0,15 \\
(0,90)\end{array}$ & $\begin{array}{l}-0,05 \\
(0,34)\end{array}$ & $\begin{array}{l}-0,16 \\
(0,96)\end{array}$ & $\begin{array}{l}-0,04 \\
(0,32)\end{array}$ & $\begin{array}{l}-0,15 \\
(0,90)\end{array}$ \\
\hline Sexe féminin & $\begin{array}{c}-0,11 \\
(2,16)^{* *}\end{array}$ & $\begin{array}{c}0,06 \\
(0,92)\end{array}$ & $\begin{array}{c}-0,09 \\
(1,73)^{*}\end{array}$ & $\begin{array}{c}0,07 \\
(1,07)\end{array}$ & $\begin{array}{c}-0,09 \\
(1,69)^{*}\end{array}$ & $\begin{array}{c}0,07 \\
(1,07)\end{array}$ \\
\hline Age & $\begin{array}{c}0,01 \\
(1,05)\end{array}$ & $\begin{array}{c}0,01 \\
(1,10)\end{array}$ & $\begin{array}{c}0,01 \\
(1,52)\end{array}$ & $\begin{array}{c}0,01 \\
(1,15)\end{array}$ & $\begin{array}{c}0,01 \\
(1,49)\end{array}$ & $\begin{array}{c}0,01 \\
(1,08)\end{array}$ \\
\hline Age $^{* * 2}$ & $\begin{array}{c}0,00 \\
(1,32)\end{array}$ & $\begin{array}{c}0,00 \\
(1,14)\end{array}$ & $\begin{array}{c}0,00 \\
(1,43)\end{array}$ & $\begin{array}{c}0,00 \\
(1,07)\end{array}$ & $\begin{array}{c}0,00 \\
(1,41)\end{array}$ & $\begin{array}{c}0,00 \\
(1,04)\end{array}$ \\
\hline Constante & $\begin{array}{c}-0,02 \\
(0,11)\end{array}$ & $\begin{array}{c}-0,20 \\
(1,08)\end{array}$ & $\begin{array}{c}-0,34 \\
(1,84)^{*}\end{array}$ & $\begin{array}{c}-0,31 \\
(1,33)\end{array}$ & $\begin{array}{c}-0,31 \\
(1,61)\end{array}$ & $\begin{array}{c}-0,30 \\
(1,29)\end{array}$ \\
\hline Observations & 837 & & 837 & & 837 & \\
\hline R2 & 0,06 & 0,00 & 0,08 & 0,01 & 0,08 & 0,01 \\
\hline Corrélation résidus & $-0,08$ & {$[$ Chi2=5,6] } & $-0,07$ & {$[$ Chi2 $=4,6]$} & $-0,07$ & {$[$ Chi2 $=4,3]$} \\
\hline
\end{tabular}

Source : Enquêtes 1-2-3, phase 3, modules qualitatifs, 1995, MADIO/INSTAT, nos propres calculs.

Notes : Valeur absolue du t-student entre parenthèses. * significatif à $10 \%$; ** significatif à $5 \%$; *** significatif à $1 \%$

Les résidus du modèle ne sont pas corrélés (avec un coefficient de -0.07 qui est non significatif, la spécification bivariée (en équations simultanées) ne se justifie pas. On obtient en fait les mêmes résultats en adoptant des équations indépendantes pour les deux variables (« en faveur de la libéralisation » et « en faveur de la démocratie »). Ce résultat confirme le fait que les opinions sur les deux options se déterminent de façon indépendante. 
Tableau 5: Facteurs explicatifs des attitudes vis-à-vis de l'État et de l'ouverture extérieure, 1998

Modèle bivarié (régression en équations simultanées)

\begin{tabular}{|c|c|c|c|c|c|c|c|c|}
\hline & $\begin{array}{c}\text { Enfaveur } \\
\text { Libéalisat'écon. }\end{array}$ & $\begin{array}{c}\text { (1) } \\
\text { Enfaveur } \\
\text { Ouveture } \\
\text { extéieure }\end{array}$ & $\begin{array}{c}\text { Enfaveur } \\
\text { Libéalisal' écon. }\end{array}$ & $\begin{array}{c}\text { (2) } \\
\text { Enfaveur } \\
\text { Ouveture } \\
\text { extéiene }\end{array}$ & $\begin{array}{c}\text { Enfaveur } \\
\text { Libézlisal écon. }\end{array}$ & $\begin{array}{c}\text { (3) } \\
\text { Enfaveur } \\
\text { Ouveture } \\
\text { extéieune }\end{array}$ & $\begin{array}{c}\text { Enfaveur } \\
\text { Libéalisat? écon. }\end{array}$ & $\begin{array}{c}\text { (4) } \\
\text { Enfaveur } \\
\text { Ouveture } \\
\text { extéieure } \\
\end{array}$ \\
\hline Edhelledepaumzéé & & & $\begin{array}{c}-0,18 \\
(13,85)^{5+1+k}\end{array}$ & $\begin{array}{c}-0,05 \\
(5,01)^{\text {s+ok }}\end{array}$ & $\begin{array}{c}-0,06 \\
(4,34)^{+\infty+2 k}\end{array}$ & $\begin{array}{c}-0,06 \\
(4,35)^{\text {thek }}\end{array}$ & $\begin{array}{c}-0,06 \\
(4,27)^{+100 *}\end{array}$ & $\begin{array}{c}-0,06 \\
(4,59)^{\text {tepek }}\end{array}$ \\
\hline Niveaudéducation & & & & & $\begin{array}{c}0,36 \\
(6,16)^{+1+2 k}\end{array}$ & $\begin{array}{c}0,00 \\
(0,05)\end{array}$ & $\begin{array}{c}0,35 \\
(6,11)^{\text {rewk }}\end{array}$ & $\begin{array}{c}0,01 \\
(0,14)\end{array}$ \\
\hline $\begin{array}{l}\text { Connaitréfome } \\
\text { fonctionpublique }\end{array}$ & & & & & $\begin{array}{c}0,08 \\
(3,74)^{\text {stoke }}\end{array}$ & $\begin{array}{l}-0,01 \\
(0,57)\end{array}$ & $\begin{array}{c}0,07 \\
(3,08)^{\text {*16*k }}\end{array}$ & $\begin{array}{l}-0,01 \\
(0,25)\end{array}$ \\
\hline Connaitprivatisation & & & & & $\begin{array}{c}0,30 \\
(13,35)^{\text {selek }}\end{array}$ & $\begin{array}{l}-0,01 \\
(0,25)\end{array}$ & $\begin{array}{c}0,27 \\
(12,22)^{\text {watk }}\end{array}$ & $\begin{array}{c}0,01 \\
(0,37)\end{array}$ \\
\hline $\begin{array}{l}\text { Victimeabsentéisme } \\
\text { desfoncionnaires }\end{array}$ & & & & & & & $\begin{array}{c}0,17 \\
(8,84)^{*+\alpha *}\end{array}$ & $\begin{array}{c}-0,07 \\
(3,83)^{\text {retek }}\end{array}$ \\
\hline Vicimecomuption & & & & & & & $\begin{array}{c}0,10 \\
(4,93)^{\text {+1ek* }}\end{array}$ & $\begin{array}{c}-0,05 \\
(2,91)^{\text {seck }}\end{array}$ \\
\hline $\begin{array}{l}\text { Aideaux licendé́s } \\
\text { desprivatisations }\end{array}$ & & & & & & & $\begin{array}{c}-0,06 \\
(1,74)^{*}\end{array}$ & $\begin{array}{c}-0,10 \\
(3,40)^{\text {retex }}\end{array}$ \\
\hline Sexeféminin & $\begin{array}{c}-0,10 \\
(4,88)^{\text {)+oke }}\end{array}$ & $\begin{array}{c}-0,02 \\
(1,14)\end{array}$ & $\begin{array}{c}-0,08 \\
(4,06)^{*+16 *}\end{array}$ & $\begin{array}{c}-0,01 \\
(0,80)\end{array}$ & $\begin{array}{l}-0,02 \\
(1,24)\end{array}$ & $\begin{array}{c}-0,01 \\
(0,85)\end{array}$ & $\begin{array}{c}-0,03 \\
(1,65)^{*}\end{array}$ & $\begin{array}{l}-0,01 \\
(0,77)\end{array}$ \\
\hline Ménagespublics & $\begin{array}{c}0,00 \\
(0,03)\end{array}$ & $\begin{array}{c}0,04 \\
(1,29)\end{array}$ & $\begin{array}{r}-0,04 \\
(0,98)\end{array}$ & $\begin{array}{c}0,03 \\
(0,94)\end{array}$ & $\begin{array}{l}-0,04 \\
(1,22)\end{array}$ & $\begin{array}{r}0,03 \\
(0,97)\end{array}$ & $\begin{array}{l}-0,03 \\
(0,99)\end{array}$ & $\begin{array}{c}0,03 \\
(0,88)\end{array}$ \\
\hline Ménagespivésfomels & $\begin{array}{l}-0,03 \\
(0,0)\end{array}$ & $\begin{array}{c}0,02 \\
(0,72)\end{array}$ & $\begin{array}{l}-0,02 \\
(0,55)\end{array}$ & $\begin{array}{c}0,02 \\
(0,79)\end{array}$ & $\begin{array}{c}0,02 \\
(0,73)\end{array}$ & $\begin{array}{c}0,02 \\
(0,75)\end{array}$ & $\begin{array}{c}0,03 \\
(0,81)\end{array}$ & $\begin{array}{c}0,02 \\
(0,74)\end{array}$ \\
\hline Ménagesinformels & $\begin{array}{c}-0,16 \\
(4,75)^{\text {;etok }}\end{array}$ & $\begin{array}{c}0,00 \\
(0,16)\end{array}$ & $\begin{array}{c}-0,09 \\
(2,69)^{1+16 k}\end{array}$ & $\begin{array}{c}0,03 \\
(0,94)\end{array}$ & $\begin{array}{l}-0,01 \\
(0,19)\end{array}$ & $\begin{array}{c}0,02 \\
(0,86)\end{array}$ & $\begin{array}{l}-0,01 \\
(0,20)\end{array}$ & $\begin{array}{c}0,02 \\
(0,81)\end{array}$ \\
\hline Age & $\begin{array}{c}0,02 \\
(5,15)^{+\infty e+k}\end{array}$ & $\begin{array}{c}0,00 \\
(0,05)\end{array}$ & $\begin{array}{c}0,02 \\
(4,68)^{+\infty 1 \% k}\end{array}$ & $\begin{array}{c}0,00 \\
(0,18)\end{array}$ & $\begin{array}{c}0,02 \\
(4,05)^{+\infty 1+k}\end{array}$ & $\begin{array}{c}0,00 \\
(0,10)\end{array}$ & $\begin{array}{c}0,02 \\
(3,90)^{\text {*kek }}\end{array}$ & $\begin{array}{c}0,00 \\
(0,03)\end{array}$ \\
\hline Age**2 & $\begin{array}{c}0,00 \\
(5,45)^{\text {tepek }}\end{array}$ & $\begin{array}{c}0,00 \\
(0,35)\end{array}$ & $\begin{array}{c}0,00 \\
(5,03)^{*+\alpha *}\end{array}$ & $\begin{array}{c}0,00 \\
(0,57)\end{array}$ & $\begin{array}{c}0,00 \\
(3,62)^{+1+1 \times k}\end{array}$ & $\begin{array}{c}0,00 \\
(0,48)\end{array}$ & $\begin{array}{c}0,00 \\
(3,41)^{1+\alpha+k}\end{array}$ & $\begin{array}{c}0,00 \\
(0,32)\end{array}$ \\
\hline Chômenus & $\begin{array}{c}0,08 \\
(1,43)\end{array}$ & $\begin{array}{r}-0,06 \\
(1,29)\end{array}$ & $\begin{array}{c}0,11 \\
(1,97)^{* i k}\end{array}$ & $\begin{array}{r}-0,05 \\
(1,11)\end{array}$ & $\begin{array}{c}0,08 \\
(1,49)\end{array}$ & $\begin{array}{r}-0,05 \\
(1,11)\end{array}$ & $\begin{array}{c}0,08 \\
(1,52)\end{array}$ & $\begin{array}{r}-0,04 \\
(0,98)\end{array}$ \\
\hline Inacifs & $\begin{array}{c}0,09 \\
(3,21)^{\text {retek }}\end{array}$ & $\begin{array}{c}0,00 \\
(0,01)\end{array}$ & $\begin{array}{c}0,08 \\
(3,02)^{\text {*1+k* }}\end{array}$ & $\begin{array}{c}0,00 \\
(0,12)\end{array}$ & $\begin{array}{c}0,03 \\
(1,29)\end{array}$ & $\begin{array}{c}0,00 \\
(0,10)\end{array}$ & $\begin{array}{c}0,04 \\
(1,61)\end{array}$ & $\begin{array}{l}-0,01 \\
(0,20)\end{array}$ \\
\hline $\begin{array}{l}\text { Religionprotestante } \\
\text { FJKM }\end{array}$ & $\begin{array}{c}0,09 \\
(4,40)^{\text {stopk }}\end{array}$ & $\begin{array}{c}-0,05 \\
(3,20)^{4+16 k}\end{array}$ & $\begin{array}{c}0,06 \\
(2,82)^{+\infty+0 k}\end{array}$ & $\begin{array}{c}-0,06 \\
(3,80)^{\text {stoke }}\end{array}$ & $\begin{array}{c}0,04 \\
(2,05)^{\text {;k }}\end{array}$ & $\begin{array}{c}-0,06 \\
(3,76)^{\text {*atok }}\end{array}$ & $\begin{array}{c}0,03 \\
(1,41)\end{array}$ & $\begin{array}{c}-0,06 \\
(3,57)^{\text {retek }}\end{array}$ \\
\hline Constante & $\begin{array}{c}-0,35 \\
(3,88)^{\text {reckek }}\end{array}$ & $\begin{array}{c}0,00 \\
(0,02)\end{array}$ & $\begin{array}{c}-0,31 \\
(3,62)^{*+\alpha *}\end{array}$ & $\begin{array}{c}0,01 \\
(0,16)\end{array}$ & $\begin{array}{c}-0,73 \\
(7,78)^{\text {seke }}\end{array}$ & $\begin{array}{c}0,01 \\
(0,16)\end{array}$ & $\begin{array}{c}-0,72 \\
(7,52)^{+16 k *}\end{array}$ & $\begin{array}{c}0,13 \\
(1,49)\end{array}$ \\
\hline Observations & & 2885 & & 2885 & & 2885 & & 2885 \\
\hline R2 & 0,05 & 0,01 & 0,11 & 0,02 & 0,21 & 0,02 & 0,24 & 0,03 \\
\hline Conélaionsrésidus & $-0,01$ & [Chi2 $=0,27]$ & $-0,03$ & {$[\mathrm{Chi} 2=3,39]$} & $-0,03$ & [Chi2=3,13] & $-0,01$ & [Chi2 $=0,58]$ \\
\hline
\end{tabular}

Source : Enquêtes 1-2-3, phase 3, modules qualitatifs, 1998, MADIO/INSTAT, nos propres calculs.

Notes : Valeur absolue du t-student entre parenthèses. * significatif à $10 \%$; ** significatif à $5 \%$; *** significatif à $1 \%$

Les résidus du modèle ne sont pas corrélés, la spécification bivariée (en équations simultanées) ne se justifie pas. On obtient en fait les

mêmes résultats en adoptant des équations indépendantes pour les deux variables ( en faveur de la libéralisation » et « en faveur de

l’ouverture »). Ce résultat confirme le fait que les opinions sur les deux options se déterminent de façon indépendante. 
L’opposition affichée à la libéralisation économique ne traduit pas une préférence systématique pour l'encadrement d'une autorité, limitant les marges de manœuvres individuelles, sachant que la libéralisation sur le front politique est revendiquée ${ }^{19}$.

Les résultats se trouvent confortés d'une année à l'autre (1995, 1998 et 2003). Pourtant, les ménages touchés par la pauvreté ne sont pas les mêmes au cours de ces différentes périodes compte tenu de l'évolution du contexte économique ${ }^{20}$. L'attitude des pauvres à l'encontre de l'économie du marché est donc bien liée au phénomène de la pauvreté et non à des caractéristiques d'un groupe spécifique d’individus dont les opinions sont arrêtées quelle que soit leur situation économique.

\subsection{Quels sont les autres facteurs explicatifs des opinions de la population ?}

Plus largement, lorsqu'on cherche à expliquer le mode de structuration des opinions sur les options économiques et politiques, il est difficile de trouver des facteurs ou caractéristiques discriminant les points de vue ${ }^{21}$. C'est particulièrement vrai en 1995 sur la démocratie, au moment où le processus de transition politique est à ses débuts (voir tableau 4).

En 1998, les femmes et les jeunes se classent avec les pauvres parmi les plus enclins à réclamer un rôle prépondérant de l'État dans la sphère économique. Sachant que ces groupes sont plus vulnérables et/ou socialement dominés, leur opinion trouve son origine dans leurs attentes : ils comptent sur les institutions publiques pour remédier à leur situation. Parallèlement, les ménages dirigés par des salariés du secteur public, plus susceptibles de subir les conséquences négatives de la libéralisation (notamment les mesures limitant les effectifs dans l'administration et les entreprises publiques) se montrent moins favorables au retrait de l'État.

Enfin, de façon cohérente, ceux qui ont été directement victimes des dysfonctionnements de l'administration (corruption ou absentéisme des fonctionnaires) se classent parmi les plus critiques à l'égard des institutions étatiques. Ils se positionnent en conséquence en faveur d'un retrait de l'État et de l'instauration d'une économie régie par les lois du marché. Ces derniers tendent cependant à s'opposer à l'ouverture extérieure du pays. Il est difficile d'expliquer ce résultat. On peut supposer que cette attitude découle du fait qu'ils associent la dégradation de l'administration à l'arrivée des étrangers.

En 2003, l'analyse distingue le point de vue à l'égard de la libéralisation, la confiance en l'efficacité des institutions étatiques, l'adhésion aux principes de la démocratie et le jugement sur la manière dont ces derniers sont appliqués (voir tableau 6a). Cette approche ajoute un éclairage nouveau sur le mode de structuration des opinions.

19 Le fait que les résidus du modèle qui traite conjointement les attitudes à l'égard de la libéralisation économique et la démocratie en 1995, ne sont pas corrélés confirme que les opinions sur les deux options se construisent de façon indépendante.

20 De manière générale, lesflux d'entrées et de sorties de la pauvreté sont importants (Herrera et Roubaud, 2003). Plus spécifiquement, à titre d'exemple, les salariés des entreprises franches ont pu bénéficier du développement et de la performance de ce secteur à la fin des années 90. Ces derniers sont, en 2002, les premières victimes de la dégradation de la situation économique avec le ralentissement des activités et la fermeture de nombreuses entreprises franches suite à la crise politique (Razafindrakoto et Roubaud, 2002b).

21

De manière générale, le pouvoir explicatif des modèles est relativement faible, avec des R2 inférieurs ou égaux à 10 \%, à l'exception du modèle sur les attitudes vis-à-vis du rôle de l'État en 1998 pour lequel le R2 atteint 24 \%. 
Tableau 6 a : Facteurs associés aux attitudes vis-à-vis de la libéralisation et de la démocratie en 2003

Modèle quadrivarié (régression en équations simultanées)

\begin{tabular}{|c|c|c|c|c|}
\hline & $\begin{array}{l}\text { (a) } \\
\text { En faveur } \\
\text { libéralisation } \\
\text { économique } \\
\end{array}$ & $\begin{array}{l}\text { (b) } \\
\text { Confiance en l'État / } \\
\text { Admin. fonctionne } \\
\text { bien }\end{array}$ & $\begin{array}{l}\text { (c) } \\
\begin{array}{l}\text { En faveur de la } \\
\text { démocratie }\end{array} \\
\end{array}$ & $\begin{array}{c}(\mathrm{d}) \\
\text { Démocratie } \\
\text { fonctionne bien dans } \\
\text { le pays }\end{array}$ \\
\hline Echelle continue pauvreté & $\begin{array}{c}-0,07 \\
(3,37)^{* * *}\end{array}$ & $\begin{array}{c}-0,06 \\
(3,02)^{* * *}\end{array}$ & $\begin{array}{c}-0,02 \\
(1,27)\end{array}$ & $\begin{array}{c}-0,03 \\
(1,66)^{*}\end{array}$ \\
\hline Niveau d'éducation & $\begin{array}{c}0,10 \\
(1,42)\end{array}$ & $\begin{array}{c}-0,21 \\
(3,19)^{* * *}\end{array}$ & $\begin{array}{l}-0,07 \\
(1,07)\end{array}$ & $\begin{array}{c}-0,21 \\
(3,98)^{* * *}\end{array}$ \\
\hline Suivent régulièrement nouvelles & $\begin{array}{c}0,09 \\
(3,47)^{* * *}\end{array}$ & $\begin{array}{c}0,01 \\
(0,45)\end{array}$ & $\begin{array}{c}0,03 \\
(1,30)\end{array}$ & $\begin{array}{l}-0,02 \\
(1,18)\end{array}$ \\
\hline Victimes corruption & $\begin{array}{c}0,06 \\
(2,06)^{* *}\end{array}$ & $\begin{array}{c}-0,04 \\
(1,37)\end{array}$ & $\begin{array}{c}-0,06 \\
(2,39)^{* *}\end{array}$ & $\begin{array}{l}-0,03 \\
(1,56)\end{array}$ \\
\hline Victimes absentéisme des fonctionnaires & $\begin{array}{c}-0,10 \\
(3,39)^{* * *}\end{array}$ & $\begin{array}{c}-0,05 \\
(1,82)^{*}\end{array}$ & $\begin{array}{c}-0,06 \\
(2,16)^{* *}\end{array}$ & $\begin{array}{c}-0,08 \\
(3,46)^{* * *}\end{array}$ \\
\hline Victimes violence & $\begin{array}{l}-0,03 \\
(1,04)\end{array}$ & $\begin{array}{l}-0,02 \\
(0,79)\end{array}$ & $\begin{array}{c}-0,06 \\
(2,26)^{* *}\end{array}$ & $\begin{array}{l}-0,03 \\
(1,12)\end{array}$ \\
\hline Estiment dégradation niveau de vie & $\begin{array}{c}0,02 \\
(0,84)\end{array}$ & $\begin{array}{c}-0,04 \\
(1,58)\end{array}$ & $\begin{array}{c}-0,05 \\
(2,20)^{* *}\end{array}$ & $\begin{array}{l}-0,01 \\
(0,55)\end{array}$ \\
\hline Participation politique (a voté aux élections) & $\begin{array}{l}-0,01 \\
(0,55)\end{array}$ & $\begin{array}{c}0,11 \\
(4,24)^{* * *}\end{array}$ & $\begin{array}{c}0,12 \\
(4,99)^{* * *}\end{array}$ & $\begin{array}{c}0,00 \\
(0,01)\end{array}$ \\
\hline Partisan politique (proche d’un parti) & $\begin{array}{c}0,06 \\
(2,73)^{* * *}\end{array}$ & $\begin{array}{c}0,10 \\
(4,25)^{* * *}\end{array}$ & $\begin{array}{c}0,10 \\
(4,66)^{* * *}\end{array}$ & $\begin{array}{c}0,05 \\
(2,73)^{* * *}\end{array}$ \\
\hline En faveur importance de la politique & $\begin{array}{c}0,08 \\
(2,43)^{* *}\end{array}$ & $\begin{array}{c}0,09 \\
(2,77)^{* * *}\end{array}$ & $\begin{array}{c}0,05 \\
(1,70)^{*}\end{array}$ & $\begin{array}{c}0,19 \\
(7,87)^{* * *}\end{array}$ \\
\hline Participat ${ }^{\circ}$ à mouvements (grève/manif.) & $\begin{array}{c}0,04 \\
(1,67)^{*}\end{array}$ & $\begin{array}{c}0,02 \\
(0,75)\end{array}$ & $\begin{array}{c}0,09 \\
(4,10)^{* * *}\end{array}$ & $\begin{array}{c}0,03 \\
(1,60)\end{array}$ \\
\hline N’appartiennent à aucune association & $\begin{array}{c}0,07 \\
(3,22)^{* * *}\end{array}$ & $\begin{array}{c}0,05 \\
(2,38)^{* *}\end{array}$ & $\begin{array}{c}0,01 \\
(0,43)\end{array}$ & $\begin{array}{c}0,01 \\
(0,52)\end{array}$ \\
\hline Primauté sentiment ethnique & $\begin{array}{c}0,12 \\
(3,94)^{* * *}\end{array}$ & $\begin{array}{c}-0,11 \\
(3,60)^{* * *}\end{array}$ & $\begin{array}{c}-0,24 \\
(8,62)^{* * *}\end{array}$ & $\begin{array}{c}0,09 \\
(3,99)^{* * *}\end{array}$ \\
\hline Société juste = Suppression inégalités & $\begin{array}{c}-0,06 \\
(2,18)^{* *}\end{array}$ & $\begin{array}{c}-0,04 \\
(1,38)\end{array}$ & $\begin{array}{l}-0,02 \\
(0,67)\end{array}$ & $\begin{array}{c}0,03 \\
(1,24)\end{array}$ \\
\hline Société juste=Reconnaissance selon mérites & $\begin{array}{l}-0,05 \\
(1,01)\end{array}$ & $\begin{array}{c}0,11 \\
(2,45)^{* *}\end{array}$ & $\begin{array}{c}0,22 \\
(4,98)^{* * *}\end{array}$ & $\begin{array}{l}-0,01 \\
(0,21)\end{array}$ \\
\hline Sexe féminin & $\begin{array}{c}-0,06 \\
(2,75)^{* * *}\end{array}$ & $\begin{array}{c}0,01 \\
(0,46)\end{array}$ & $\begin{array}{c}0,01 \\
(0,69)\end{array}$ & $\begin{array}{c}0,04 \\
(2,57)^{* *}\end{array}$ \\
\hline Age & $\begin{array}{c}0,01 \\
(2,42)^{* *}\end{array}$ & $\begin{array}{c}0,00 \\
(1,02)\end{array}$ & $\begin{array}{c}0,00 \\
(1,19)\end{array}$ & $\begin{array}{c}0,00 \\
(1,02)\end{array}$ \\
\hline Age**2 & $\begin{array}{c}0,00 \\
(2,50)^{* *}\end{array}$ & $\begin{array}{c}0,00 \\
(0,75)\end{array}$ & $\begin{array}{c}0,00 \\
(1,15)\end{array}$ & $\begin{array}{c}0,00 \\
(0,79)\end{array}$ \\
\hline Constante & $\begin{array}{c}-0,24 \\
(2,13)^{* *}\end{array}$ & $\begin{array}{c}0,00 \\
(0,00)\end{array}$ & $\begin{array}{c}-0,17 \\
(1,69)^{*}\end{array}$ & $\begin{array}{c}0,09 \\
(1,03)\end{array}$ \\
\hline Observations & 2522 & 2522 & 2522 & 2522 \\
\hline R2 & 0,06 & 0,05 & 0,10 & 0,06 \\
\hline
\end{tabular}

Source : Enquêtes 1-2-3, phase 3, modules qualitatifs, 2002-2003, INSTAT, nos propres calculs.

Valeur absolue du t-student entre parenthèses. * significatif à $10 \%$; ** significatif à $5 \%$; *** significatif à $1 \%$ 
Tableau 6 b : Corrélation de la matrice des résidus du modèle quadrivarié en 2003

\begin{tabular}{|c|c|c|c|c|}
\hline & $\begin{array}{l}\text { (a) } \\
\text { En faveur } \\
\text { libéralisation écon. }\end{array}$ & $\begin{array}{l}\text { (b) } \\
\text { Confiance en l’État } \\
\text { / fonctionnement } \\
\text { adm. }\end{array}$ & $\begin{array}{l}\text { (c) } \\
\text { En faveur de la } \\
\text { démocratie }\end{array}$ & $\begin{array}{l}\text { (d) } \\
\text { Fonctionnement } \\
\text { démocratie dans } \\
\text { pays } \\
\end{array}$ \\
\hline $\begin{array}{l}\text { En faveur libéralisation économique } \\
\text { Confiance en l'État / fonctionnement } \\
\text { adm. }\end{array}$ & 1,00 & 1,00 & & \\
\hline $\begin{array}{l}\text { En faveur de la démocratie } \\
\text { Fonctionnement démocratie dans le } \\
\text { pays }\end{array}$ & 0,05 & 0,30 & 1,00 & 1,00 \\
\hline
\end{tabular}

Test d'indépendance de Breusch-Pagan : chi2 (6) = 312.096, $\operatorname{Pr}=0.0000$

En premier lieu, comme en 1995 et en 1998, les pauvres manifestent leur réticence face à la libéralisation économique. Si les pauvres expriment plus de méfiance vis-à-vis de l'État en 2003, cette défiance ne les conduit pas pour autant à revendiquer l'instauration de l'économie de marché et à réclamer le désengagement de l'État. Parallèlement, ils tendent à être plus critiques sur la manière dont la démocratie fonctionne dans le pays, mais ne se distinguent pas du reste de la population en termes d'adhésion aux principes démocratiques.

En deuxième lieu, lorsqu'on explore les autres facteurs pouvant expliquer les attitudes, il apparaît que l'insertion dans la vie politique joue favorablement sur le soutien au processus de double transition politique et économique - en œuvre. Elle conduit aussi à juger positivement le fonctionnement actuel des institutions. Ainsi, ceux qui votent aux élections sont favorables à la démocratie et font confiance à l'État. Les sympathisants des partis politiques ont une opinion positive sur les quatre points: la libéralisation, le fonctionnement de l'État, la démocratie et son fonctionnement. Ce résultat est logique dans la mesure où ceux qui participent à la vie politique ont contribué aux prises de décision, font d'une certaine manière partie du système, et sont sans doute les mieux à même d'en tirer profit ${ }^{22}$.

Ainsi, les plus critiques à l'égard des processus en cours de libéralisation et de démocratisation se trouvent marginalisés ou se marginalisent de la vie politique et ont par conséquent peu de moyens d'influer sur les décisions. Il s'agit de ceux qui n’ont pas voté aux élections, ceux qui estiment qu'aucun parti ne représente leurs aspirations et ceux qui n’ont participé à aucun mouvement organisé de protestation.

En troisième lieu, on note une corrélation entre la confiance à l'égard de l'État et l'adhésion aux principes de la démocratie ${ }^{23}$. Une opinion négative sur le fonctionnement actuel des institutions étatiques conduit ainsi à douter de la pertinence de la transition démocratique. Le cas spécifique des victimes de l'absentéisme des fonctionnaires permet d'illustrer ce constat. Ayant subi les défaillances de l'administration, ces derniers ne font pas confiance aux institutions publiques en général et condamnent à la fois les deux processus en cours de transition - politique et économique ${ }^{24}$. Plus largement, le fait de vivre une expérience négative (corruption, délai dans les démarches administratives, violence, dégradation du niveau de vie) amène à une moindre prédisposition à adhérer à l'« idéal démocratique ».

22 Soulignons que les partis qui sont dans l'opposition à Madagascar ne se démarquent pas du pouvoir par des options économiques ou politiques différentes. Les partis, dépourvus d'idéologie bien tranchée et stable, sont versatiles avec des changements fréquents de camp ou d'alliance au gré des intérêts de leurs dirigeants (voir Roubaud, 2000).

23 La corrélation entre les deux variables est assez forte puisque le coefficient est significatif et vaut 0,34 (voir tableau A3 en annexe). On constate également une corrélation des résidus du modèle multivarié (avec un coefficient égal à 0,30 ; voir tableau $6 \mathrm{~b}$ ). Des carcatéristiques inobservables dans le modèle jouent ainsi conjointement et dans le même sens sur la confiance à l'égard de l'État et l'adhésion à la démocratie.

24 Rappelons que les victimes des dysfonctionnements de l’administration se montraient favorables à la libéralisation économique en 1998, espérant donc que cette option accroîtrait l'efficacité du secteur public. Ce changement dans la façon dont les victimes se positionnent cinq années plus tard pourrait s'expliquer par le constat que la transition économique peine à conduire à l'amélioration escomptée au fil du temps. 
Des mécanismes pouvant mener à des opinions radicales à l'encontre de la démocratie sont ainsi mis en lumière. Une telle attitude semble découler d'un profond dépit vis-à-vis des systèmes politiques et économiques existants et d'une perte de confiance dans les institutions publiques les mettant en œuvre.

Ces résultats tendent à conforter la thèse selon laquelle la persistance de conditions de vie difficiles risque de réduire le soutien au processus de démocratisation. Mais ils montrent surtout que le fait d'instaurer les conditions la démocratie (élections, liberté d'expression, égalité devant la loi, etc.) ne suffit pas si les institutions chargées de veiller à leur application ne sont pas efficaces et dignes de confiance.

Par ailleurs, l'analyse permet d'identifier des facteurs qui éclairent sur la perception, les attentes et les logiques d’un certain nombre de groupes spécifiques. Ainsi, les résultats montrent que :

- conformément au résultat que l'on pouvait attendre, ceux qui dénoncent les inégalités comme une forme d'injustice et réclament leur élimination manifestent des réticences à l'égard de la libéralisation ;

- de façon tout aussi prévisible, la démocratie est préconisée par ceux qui revendiquent une " reconnaissance selon les mérites ", les régimes autoritaires ayant tendance à favoriser les groupes proches du pouvoir indépendamment de leurs performances ;

- les ménages dépourvus ou faiblement dotés en capital social accordent de façon logique leur confiance à l'État, seule entité qu'ils jugent susceptible de veiller à leurs intérêts. Toutefois, ils se positionnent en même temps en faveur de la libéralisation économique. N'appartenant à aucun réseau, ils prônent sans doute des valeurs plus individualistes et considèrent qu'un système régulé par le marché est le mieux à même de leur offrir des opportunités de réussir du point de vue économique ;

- enfin, les individus qui mettent en avant leur appartenance ethnique, au détriment du sentiment national, sont critiques et affichent une méfiance vis-à-vis de l'État. Ils se classent parmi les anti-démocrates. Le repli ethnique est donc lié à une absence de confiance à l'égard des institutions publiques nationales. La causalité peut jouer dans les deux sens : d'un côté, ceux qui font primer leur identité ethnique sont naturellement amenés à se méfier des institutions qui sont censées veiller avant tout aux intérêts de la nation ; de l'autre, la perte de confiance vis-à-vis de l’État peut conduire au développement de logiques communautaires.

\section{CONCLUSION}

Une importance croissante est accordée à la gouvernance et à la démocratie dans les stratégies de développement. La participation de la population, et en particulier des pauvres, à l'élaboration des décisions politiques et économiques est considérée comme un des éléments-clefs pour favoriser l'efficacité des stratégies mises en œuvre. Pourtant, les connaissances sur le point de vue des pauvres sont encore limitées dans les pays concernés. Cette étude témoigne de l'intérêt de la disponibilité d'informations détaillées sur l'opinion de la population suivant leurs caractéristiques économiques et socio-démographiques.

L'analyse montre que les pauvres se caractérisent par leur réticence vis-à-vis de la libéralisation économique. En revanche, leur adhésion à la démocratie est aussi massive que celle de l'ensemble de leurs concitoyens. Ces résultats sont robustes puisqu'on les retrouve pour différentes années, malgré des bouleversements du contexte économique et politique entraînant des changements dans la composition de la population pauvre.

Par ailleurs, on démontre que l'attitude des pauvres ne s'explique pas uniquement par leur faible niveau d'instruction ou d'information. Leur position contre la libéralisation - privatisation et ouverture extérieure - traduit leur appréciation positive du rôle que joue ou peut jouer l’État en faveur des plus pauvres, malgré la déficience des institutions étatiques. Comme les pauvres expriment autant que le reste de la population leur aspiration à la démocratie, leur point de vue ne peut s’interpréter comme 
une appréhension de la nouveauté ou du changement, ni comme une aversion contre les risques induits par un accroissement des marges de liberté individuelle.

Finalement, la thèse selon laquelle les plus défavorisés ont besoin d'un État qui les protège et prend mieux en compte leurs intérêts peut être confortée et soutenue à partir de deux arguments qui découlent de l'analyse :

- d'une part, l'État - et non le marché - est jugé plus apte à garantir l'objectif d'équité. L'existence de conflits d'intérêt au sein de la population, en particulier entre les riches et les plus démunis, ne peut se résoudre que par la médiation d'institutions qui visent l'intérêt du plus grand nombre ;

- d'autre part, le processus de démocratisation, auquel les pauvres adhèrent, n'est pas compatible avec un affaiblissement de l'État. La démocratie n'est effective que si des institutions dignes de confiance veillent à l'application de ses principes de base (en particulier l'égalité devant la loi et la participation de tous les citoyens). La consolidation de la démocratie ne peut se faire sans un État capable d'assurer le fonctionnement efficace des institutions. 


\section{REFERENCES BIBLIOGRAPHIQUES}

Afrobarometer (2003), « Popular Attitudes to Democracy, Selected African Countries, 1999-2001; Popular Attitudes to Markets, Selected African Countries, 1999-2000 », Afrobarometer, [http://www.afrobarometer.org/survey.html]

Alvare M., Cheibub J.A., Limongi F., Przeworski A., (2000), Democracy and Development: Political Institutions and Well-Being in the World, 1950-1990. Cambridge: Cambridge University Press.

Banque mondiale (2001), Rapport sur le développement dans le monde 2000/01: combattre la pauvreté, Editions Eska, Paris (version anglaise publiée en 2000 par Oxford University Press, New York).

Banque mondiale (2002), World Development Report 2002: Building Institutions for Markets, Banque Mondiale, Washington.

Bhagwati J.N. (2002), « Democracy and Development: Cruel Dilemma or Symbiotic Relationship? » Review of Development Economics, 6(2), pp.151-162.

Bratton M., Lewis P., Gyimah-Boadi E. (2000), «Attitudes to Democracy and Markets in Ghana », Afrobarometer Working Papers, $n^{\circ} 2$.

Bratton M., Mattes R. (2000), «Democratic and Market Reforms in Africa: What «the People » Say », Afrobarometer Working Papers, ${ }^{\circ} 5$.

Bratton M., Davids Y.D., Mattes R., (2003), « Poverty, survival and democracy in Southern Africa », Afrobarometer Working Papers, n²3, January.

Bratton M. Logan C.J., Muwanga N., Sentamu R., (2003), «Insiders and Outsiders: Varying Perceptions of Democracy and Governance in Uganda », Afrobarometer Working Papers, $n^{\circ} 27$.

Bratton M. Mattes R., (2003), «Learning about democracy in Africa: Awareness, performance, and experience », Afrobarometer Working Papers, $n^{\circ} 31$, October.

Bratton M., Mattes R. (2003), «Support for Economic Reform? Popular Attitudes in Southern Africa », World Development Vol.31, No.2, pp.303-323.

Chambers R., Narayan D., Shah M.K., Petesch P. (2000a), Crying out for Change (Voices of the poor, volume 2), Oxford University Press, World Bank.

Cling J.-P., Razafindrakoto M., Roubaud F., (dir.) (2002), Les nouvelles stratégies internationales de lutte contre la pauvreté, Economica, Paris.

Dalton R.J., Ong N.T. (2002), « Democracy and markets: Citizen Values in the Pacific Rim Region ». Paper presented at the Hawaï International Conference on the Social Sciences, Honolulu, June 11-15, 2002.

Dalton R.J., Ong N.T. (2003), « Authority Orientations and Democratic Attitudes in East Asia: A Test of the « Asian Values » Hypothesis », Centre for the Study of Democracy, University of California, Irvine (www.democ.uci.edu). Presented at the conference « How People View Democracy: Public Opinion in New Democracies », at the Center for Democracy, Development and the Rule of the Law, Stanford University.

Elster, J. (1993), "The necessity and impossibility of simultaneous economic and political reform », in D. Greenberg (ed.), Constitutional democracy: Transitions in the contemporary world, pp. 268-279, New York: Oxford University Press.

Feng, Y. (2003), Democracy, Governance, and Economic Performance. Theory and Evidence, MIT Press, Cambridge, Massachussetts.

Graham C., Pettinato S. (2001), « Happiness, Markets and Democracy: Latin America in Comparative Perspective », Journal of Happiness Studies, vol.2, pp.237-268. 
Graham C., Sukhtankar S. (2002), «Is Economic Crisis Reducing Support for Markets and Democracy in Latin America? Some evidence from Economics of Happiness », Center on Social and Economic Dynamics Working Paper Series, The Brookings Institution.

Haggard S., Webb S.B. (1994), Voting for Reform: Democracy, Political Liberalization, and Economic Adjustment, Oxford University Press, World Bank, July.

Harrison L., Huntington, S. (eds.) (2000), Culture matters: How values shape human progress, New York: Basic Books.

Herrera J., Roubaud F. (2003), «Dynamique de la pauvreté urbaine au Pérou et à Madagascar 1997-1999 : une analyse sur données de panel », Document de travail DIAL DT/2003/03, mai.

Inglehart R. (1993), La transition culturelle dans les sociétés industrielles avancées, Economica.

Inglehart R. (1997), Modernization and Postmodernization. Cultural, economic and political change in 43 societies, Princeton University Press, Princeton, 453p.

Iqbal F., You J.-I. (2001), Democracy, Market Economics, and Development: An Asian Perspective, World Bank, May.

Kaufmann D., Kraay A. (2002), "Growth without Governance », World Bank Policy Research Working Paper n²928, November.

Koch-Schulte S., Narayan D., Patel R., Rademacher A., Schafft K., (2000b), Can anyone hear us? Voices from 47 countries (Voices of the poor, volume 1), Oxford University Press, World Bank.

Kolstad I., Stern N., Tungodden B. (eds.) (2004), Toward Pro-Poor Policies - Aid, Institutions and Globalization, World Bank/Oxford University Press, New York.

Lindblom C. (1977), Politics and Markets. New York: Basic Books.

Lokshin M., Ravallion M. (1998), « Subjective Economic Welfare ». Policy Research Working Paper, World Bank, Washington D.C. 38p.

Lollivier S., Verger D. (1997) : " Pauvreté d'existence, monétaire ou subjective sont distinctes », Economie et Statistique, n³08-309-310, pp 113-142.

Présenté en 2000 lors de la conférence «Comparaisons internationales de la pauvreté », Bratislava, SUSR-INSEE-EUROSTAT (pp.339-371 dans les Actes de la conférence).

Ramamonjisoa J. (2002), « Les relations entre ethnies à Madagascar: une problématique souvent mal posée », Afrique contemporaine n²02-203, avril-sept. pp.55-71.

Ramilison E. (2003), « Impact de la crise politico-économique de 2002 sur l'emploi et les conditions d'activité des ménages de l'agglomération d'Antananarivo ", INSTAT/MADIO, Antananarivo, Madagascar.

Rannan-Eliya R. P. (1996), "Results from the IPS/Harvard Public Opinion Poll on User Fees », Health Policy Programme Occasional Paper 2. Colombo: Institute of Policy Studies.

Razafindrakoto M., Roubaud F. (1996), « Ce qu’attendent les Tananariviens de la réforme de l’État et de l'économie », Revue Politique Africaine, n²61, Karthala, Paris, pp.54-72.

Razafindrakoto M., Roubaud F. (2002a), « Pensent-ils différemment ? La voix des pauvres à travers les enquêtes statistiques ", in J.-P. Cling, Razafindrakoto M., Roubaud F., (dir.), Les nouvelles stratégies internationales de lutte contre la pauvreté, Economica, Paris, pp.139-161.

Razafindrakoto M., Roubaud F. (2002b), «Madagascar à la croisée des chemins : la croissance durable est-elle possible ? », Afrique contemporaine ${ }^{\circ}$ 202-203, avril-sept. pp.75-92.

Razafindrakoto M., Roubaud F. (2004), «Les multiples facettes de la pauvreté dans un pays en développement : Le cas de la capitale malgache », Economie et Statistique, INSEE.

Richard J.L. (2000) : « Les valeurs économiques : entre libéralisme et interventionnisme », in Bréchon P., Laurent A., Perrineau P. (dirs 2000), Les cultures politiques des français, Presse de Sciences Po, Paris, pp. 91-110. 
Roubaud F. (2000), Identités et transition démocratique: l'exception malgache? L’Harmattan/Tsipika, Paris, Antananarivo, 256p.

Sahn D.E. et Stifel D.C. (2000), " Poverty Comparisons over Time and Across Countries in Africa », World Development, 28(1), 2123-55, December.

Sandbrook R. (2000), "Citizenship, Rights and Poverty. Narrowing the Gap between Theory and Practice ", Paper Presented to the International IDEA Democracy Forum, "Democracy and Poverty: A Missing Link?", Stockholm, Sweden, June $8^{\text {th }}-9^{\text {th }}$.

Seddon D., Walton J. (1994), Free Markets and Food Riots: The politics of global adjustment. London: Blackwell.

Varshney A. (1999) «Democracy and Poverty », Paper for the Conference on World Development Report 2000, organized by the U.K. Department for International Development and the Institute of Development Studies, Sussex, August 15-16. 


\section{ANNEXES}

\section{Annexe 1: Matrice de corrélations}

Tableau A 1 : Matrice des corrélations entre la pauvreté et les positions vis-à-vis de la démocratie et la libéralisation en 1995

\begin{tabular}{|c|c|c|c|c|}
\hline & $\begin{array}{c}\text { En faveur } \\
\text { libéralisation écon. }\end{array}$ & $\begin{array}{l}\text { En faveur } \\
\text { démocratie }\end{array}$ & Niveau de pauvreté & Niveau d'éducation \\
\hline $\begin{array}{l}\text { En faveur libéralisation } \\
\text { économique }\end{array}$ & 1.00 & & & \\
\hline En faveur démocratie & $\begin{array}{l}0.08^{*} \\
(0.03)\end{array}$ & 1.00 & & \\
\hline Niveau de pauvreté & $\begin{array}{l}-0.22^{*} \\
(0.00)\end{array}$ & $\begin{array}{c}0.01 \\
(0.81)\end{array}$ & 1.00 & \\
\hline Niveau d'éducation & $\begin{array}{l}0.21 * \\
(0.00)\end{array}$ & $\begin{array}{c}0.02 \\
(0.50)\end{array}$ & $\begin{array}{l}-0.43^{*} \\
(0.00)\end{array}$ & 1.00 \\
\hline
\end{tabular}

Source : Enquêtes 1-2-3, phase 3, modules qualitatifs, 1995, MADIO/INSTAT, nos propres calculs.

Notes : Test de significativité entre parenthèses.

Tableau A 2 : Matrice des corrélations entre la pauvreté et les positions vis-à-vis de la libéralisation et l'ouverture extérieure en 1998

\begin{tabular}{|c|c|c|c|c|}
\hline & $\begin{array}{c}\text { En faveur } \\
\text { libéralisation écon. }\end{array}$ & $\begin{array}{c}\text { En faveur } \\
\text { ouverture ext. }\end{array}$ & Niveau de pauvreté & Niveau d'éducation \\
\hline $\begin{array}{l}\text { En faveur libéralisation } \\
\text { économique }\end{array}$ & 1.00 & & & \\
\hline En faveur ouverture extérieure & $\begin{array}{l}-0.01 \\
(0.56)\end{array}$ & 1.00 & & \\
\hline Niveau de pauvreté & $\begin{array}{l}-0.29 * \\
(0.00)\end{array}$ & $\begin{array}{l}-0.09 * \\
(0.00)\end{array}$ & 1.00 & \\
\hline Niveau d'éducation & $\begin{array}{l}0.31^{*} \\
(0.00)\end{array}$ & $\begin{array}{l}0.027 \\
(0.15)\end{array}$ & $\begin{array}{c}-0.51^{*} \\
(0.00)\end{array}$ & 1.00 \\
\hline
\end{tabular}

Source : Enquêtes 1-2-3, phase 3, modules qualitatifs, 1998, MADIO/INSTAT, nos propres calculs.

Notes : Test de significativité entre parenthèses.

Tableau A 3 : Matrice des corrélations entre la pauvreté et les positions vis-à-vis de la démocratie et la libéralisation en 2002

\begin{tabular}{|c|c|c|c|c|c|c|}
\hline & $\begin{array}{c}\text { (a) } \\
\text { En faveur } \\
\text { libéralisation écon. }\end{array}$ & $\begin{array}{l}\text { (b) } \\
\text { Confiance en l'État } \\
\text { / fonctionnement } \\
\end{array}$ & $\begin{array}{c}\text { (c) } \\
\text { En faveur de la } \\
\text { démocratie }\end{array}$ & $\begin{array}{c}(\mathrm{d}) \\
\text { Fonctionne- } \\
\text { ment } \\
\text { démocratie } \\
\end{array}$ & $\begin{array}{c}\text { Niveau de } \\
\text { pauvreté }\end{array}$ & $\begin{array}{c}\text { Niveau } \\
\text { d'éducation }\end{array}$ \\
\hline En faveur libéralisation écon. & 1,00 & & & & & \\
\hline Confiance État / fonctionn. adm. & $\begin{array}{c}0,00 \\
(0,98)\end{array}$ & 1,00 & & & & \\
\hline En faveur de la démocratie & $\begin{array}{l}0,05^{*} \\
(0,01)\end{array}$ & $\begin{array}{l}0,34^{*} \\
(0,00)\end{array}$ & 1,00 & & & \\
\hline Fonctionnement démocratie pays & $\begin{array}{l}0,05^{*} \\
(0,01)\end{array}$ & $\begin{array}{l}0,19 * \\
(0,00)\end{array}$ & $\begin{array}{c}0,00 \\
(1,00)\end{array}$ & 1,00 & & \\
\hline Niveau de pauvreté & $\begin{array}{l}-0,14 * \\
(0,00)\end{array}$ & $\begin{array}{l}-0,04 * \\
(0,03)\end{array}$ & $\begin{array}{l}-0,04 * \\
(0,05)\end{array}$ & $\begin{array}{c}0,02 \\
(0,22)\end{array}$ & 1,00 & \\
\hline Niveau d'éducation & $\begin{array}{l}0,12^{*} \\
(0,00)\end{array}$ & $\begin{array}{l}-0,03 \\
(0,21)\end{array}$ & $\begin{array}{c}0,01 \\
(0,59)\end{array}$ & $\begin{array}{l}-0,08^{*} \\
(0,00)\end{array}$ & $\begin{array}{l}-0,58^{*} \\
(0,00)\end{array}$ & 1,00 \\
\hline
\end{tabular}

Source : Enquêtes 1-2-3, phase 3, modules qualitatifs, 2002-2003, INSTAT, nos propres calculs. Notes : Test de significativité entre parenthèses. 


\section{Annexe 2: Liste des variables mobilisées pour les ACM}

\section{En 1995 :}

\section{Libéralisation économique et rôle de l'Etat}

1. En faveur de la libéralisation économique

En faveur de l'économie administrée

2. En faveur de l'intervention de l'État dans la sphère économique

L'État doit laisser place au secteur privé et jouer le rôle de simple arbitre

3. En faveur de la privatisation de toutes entreprises

En faveur de la privatisation de certaines entreprises

Contre les privatisations

\section{Démocratisation}

4. Favorable à la démocratie

Contre la démocratie

5. Estime qu'il faudrait plus de démocratie

Estime que l'on n'a pas besoin de plus de démocratie

\section{En 1998 :}

\section{Libéralisation économique et rôle de l'Etat}

1. En faveur de la privatisation des entreprises

Contre les privatisations

2. Entreprises publiques bien gérées

Entreprises publiques mal gérées

3. Opérateurs privés plus efficaces

Opérateurs privés moins efficaces

4. Administration efficace

Administration non efficace

5. Confiance totale à l'égard de l'administration

Confiance élevée à l'égard de l'administration

Pas du tout confiance à l'égard de l'administration

6. Les fonctionnaires travaillent beaucoup

Les fonctionnaires ne travaillent pas assez

7. Administration politisée

Administration non politisée

\section{Ouverture extérieure}

8. Priorités aux malgaches lors des privatisations Malgaches non prioritaires

9. Intérêts économiques étrangers trop importants

Poids faibles des intérêts étrangers

10. Les étrangers sont trop nombreux Le nombre d'étrangers est faible

11. En faveur de réformes autorisant les étrangers à acheter des terrains

En faveur des mesures autorisant la signature de bail emphytéotique avec un étranger $\mathrm{Ni}$ achat ni bail emphytéotique pour les étrangers 


\section{En 2003 :}

\section{Libéralisation économique et rôle de l'Etat}

1. En faveur de la libéralisation économique

En faveur de l'économie administrée

2. En faveur de l'intervention de l'État dans la sphère économique

L’État doit laisser place au secteur privé et jouer le rôle de simple arbitre

3. En faveur de la privatisation de toutes entreprises

En faveur de la privatisation de certaines entreprises

Contre les privatisations

4. Favorable à l'école publique pour tous

Favorable à l'existence à la fois de l'école publique et privée

Pour le retrait total de l'État du système éducatif

5. Estime que l'administration fonctionne bien

Estime que l'administration fonctionne mal

6. A confiance à l'égard de l'administration

n’a pas du tout confiance à l'égard de l'administration

\section{Démocratisation}

7. Favorable à la démocratie

Contre la démocratie

8. Estime que la démocratie est mieux que tout autre type de régime

Estime que la démocratie n'est pas le meilleur type de régime

9. La démocratie est source de beaucoup de problèmes (économiques, troubles politiques, maintien de l'ordre)

La démocratie crée quelques problèmes

La démocratie ne crée pas de problèmes

10. Juge plus favorablement les systèmes dirigés par un homme fort (sans Parlement ni élections) ou dirigés par l'armée ou par des experts Juge défavorablement ces systèmes politiques

11. Juge favorablement un système politique démocratique Juge négativement un système politique démocratique

12. La démocratie fonctionne bien dans le pays La démocratie fonctionne assez bien dans le pays La démocratie fonctionne mal dans le pays

13. Les principes de la démocratie (liberté d'expression, égalité devant la loi, liberté politique, élections libres et transparentes, liberté de voyager, liberté de religion) sont bien respectés dans le pays

Ces principes sont moyennement respectés

Ces principes sont très peu respectés 